\title{
SYMPLECTIC CHAIN COMPLEX AND REIDEMEISTER TORSION OF COMPACT MANIFOLDS
}

\author{
YAŞAR SÖZEN
}

\begin{abstract}
Using symplectic complex, this article proves a formula for computing the Reidemeister torsion of even dimensional oriented closed connected manifolds. Moreover, it presents applications to Riemann surfaces and Grassmannians.
\end{abstract}

\section{Introduction}

Reidemeister torsion is a topological invariant and was introduced by Reidemeister in 1935. Up to PL equivalence, he classified the lens spaces $S^{3} / \Gamma$, where $\Gamma$ is a finite cyclic group of fixed point free orthogonal transformations [20]. In [8], Franz extended the Reidemeister torsion and classified the higher dimensional lens spaces $S^{2 n+1} / \Gamma$, where $\Gamma$ is a cyclic group acting freely and isometrically on the sphere $\mathrm{S}^{2 n+1}$.

In 1964, the results of Reidemeister and Franz were extended by de Rham to spaces of constant curvature +1 [7]. Kirby and Siebenmann proved the topological invariance of the Reidemeister torsion for manifolds in 1969 [12]. Chapman proved invariance for arbitrary simplicial complexes [5], [6]. Hence, the classification of lens spaces of Reidemeister and Franz was actually topological (i.e., up to homeomorphism).

Using the Reidemeister torsion, Milnor disproved Hauptvermutung in 1961. He constructed two homeomorphic but combinatorially distinct finite simplicial complexes. He identified in 1962 the Reidemeister torsion with the Alexander polynomial which plays an important role in knot theory and links [16], [18].

In the paper [22], we explained the claim mentioned in [27, p. 187] about the relation between a symplectic chain complex with $\omega$-compatible bases and its Reidemeister torsion (Theorem 1.7). Moreover, we applied Theorem 1.7 to the chain-complex

$$
0 \longrightarrow C_{2}\left(\Sigma_{g} ; \mathrm{Ad}_{\varrho}\right) \stackrel{\partial_{2} \otimes \mathrm{id}}{\longrightarrow} C_{1}\left(\Sigma_{g} ; \mathrm{Ad}_{\varrho}\right) \stackrel{\partial_{1} \otimes i d}{\longrightarrow} C_{0}\left(\Sigma_{g} ; \mathrm{Ad}_{\varrho}\right) \longrightarrow 0,
$$


where $\Sigma_{g}$ is a compact Riemann surface of genus $g>1$, where $\partial$ is the usual boundary operator, and where $\varrho: \pi_{1}\left(\Sigma_{g}\right) \rightarrow \mathrm{PSL}_{2}(\mathrm{R})$ is a discrete and faithful representation of the fundamental group $\pi_{1}\left(\Sigma_{g}\right)$ of $\Sigma_{g}$ [22].

In the present article, oriented closed connected $2 m$-manifolds $(m \geq 1)$ are considered and the following formula for computing the Reidemeister torsion of them is proved. Namely,

THEOREM 0.1. Let $M$ be an oriented closed connected $2 m$-manifold $(m \geq$ 1). For $p=0, \ldots, 2 m$, let $\mathbf{h}_{p}$ be a basis of $H_{p}(M)$. Then, the Reidemeister torsion of $M$ satisfies the following formula:

$$
\left|\mathbf{T}\left(M,\left\{\mathbf{h}_{p}\right\}_{0}^{2 m}\right)\right|=\prod_{p=0}^{m-1}\left|\operatorname{det} H_{p, 2 m-p}(M)\right|^{(-1)^{p}}{\sqrt{\left|\operatorname{det} H_{m, m}(M)\right|}}^{(-1)^{m}},
$$

where det $H_{p, 2 m-p}(M)$ is the determinant of the matrix of the intersection pairing $(\cdot, \cdot)_{p, 2 m-p}: H_{p}(M) \times H_{2 m-p}(M) \rightarrow \mathrm{R}$ in bases $\mathbf{h}_{p}, \mathbf{h}_{2 m-p}$.

Throughout the paper, by manifold we mean smooth manifold.

It is well known that Riemann surfaces and Grassmannians have many applications in a wide range of mathematics such as topology, differential geometry, algebraic geometry, symplectic geometry, and theoretical physics (see, e.g., [1]-[4], [9], [10], [13], [14], [22]-[27], and the references therein). We also apply Theorem 0.1 to Riemann surfaces and Grassmannians.

The content of the paper is as follows. In $\S 1$, we provide the basic definitions and facts about the Reidemeister torsion of a general chain complex. Moreover, we explain symplectic chain complexes. §2 concerns the Reidemeister torsion of a manifold. We explain in $\$ 3$ the symplectic chain complex associated to a $2 m$-manifold with $m$ odd. Furthermore, the proof of Theorem 0.1 is given. As applications, Theorem 0.1 is applied in $\S 4$ to Riemann surfaces and Grassmanians.

\section{Reidemeister torsion of a chain complex}

In this section, the required definitions and the basic facts about the Reidemeister torsion are given. Detailed proofs and more information can be found in [19], [22], [27], and the references therein.

We shall reserve $F$ to denote the field of real $R$ or complex $C$ numbers. Let

$$
\left(C_{*}, \partial_{*}\right)=\left(C_{n} \stackrel{\partial_{n}}{\longrightarrow} C_{n-1} \rightarrow \cdots \rightarrow C_{1} \stackrel{\partial_{1}}{\longrightarrow} C_{0} \rightarrow 0\right)
$$

be a chain complex of finite dimensional vector spaces over F. Let $H_{p}\left(C_{*}\right)=$ $Z_{p}\left(C_{*}\right) / B_{p}\left(C_{*}\right)$ denote the $p$-th homology of $C_{*}$, where $B_{p}\left(C_{*}\right)=\operatorname{Im}\left\{\partial_{p+1}\right.$ : $\left.C_{p+1} \rightarrow C_{p}\right\}$, and $Z_{p}\left(C_{*}\right)=\operatorname{ker}\left\{\partial_{p}: C_{p} \rightarrow C_{p-1}\right\}$. 
Clearly, we have the following short-exact sequences: $0 \rightarrow Z_{p}\left(C_{*}\right) \rightarrow$ $C_{p} \rightarrow B_{p-1}\left(C_{*}\right) \rightarrow 0$ and $0 \rightarrow B_{p}\left(C_{*}\right) \rightarrow Z_{p}\left(C_{*}\right) \rightarrow H_{p}\left(C_{*}\right) \rightarrow 0$. Assume that $\mathbf{b}_{p}, \mathbf{h}_{p}$ are bases of $B_{p}\left(C_{*}\right), H_{p}\left(C_{*}\right)$, respectively. Assume also that $\ell_{p}: H_{p}\left(C_{*}\right) \rightarrow Z_{p}\left(C_{*}\right), s_{p}: B_{p-1}\left(C_{*}\right) \rightarrow C_{p}$ are sections of $Z_{p}\left(C_{*}\right) \rightarrow$ $H_{p}\left(C_{*}\right), C_{p} \rightarrow B_{p-1}\left(C_{*}\right)$, respectively. Then, we obtain a new basis of $C_{p}$, namely $\mathbf{b}_{p} \oplus \ell_{p}\left(\mathbf{h}_{p}\right) \oplus s_{p}\left(\mathbf{b}_{p-1}\right)$.

DEFINITION 1.1. Let

$$
C_{*}: C_{n} \stackrel{\partial_{n}}{\longrightarrow} C_{n-1} \rightarrow \cdots \rightarrow C_{1} \stackrel{\partial_{1}}{\longrightarrow} C_{0} \rightarrow 0
$$

be a chain complex of finite dimensional vector spaces over F. For $p=$ $0, \ldots, n$, let $\mathbf{c}_{p}, \mathbf{b}_{p}, \mathbf{h}_{p}$ be bases of $C_{p}, B_{p}\left(C_{*}\right), H_{p}\left(C_{*}\right)$, respectively, and let $\ell_{p}: H_{p}\left(C_{*}\right) \rightarrow Z_{p}\left(C_{*}\right), s_{p}: B_{p-1}\left(C_{*}\right) \rightarrow C_{p}$ be sections of $Z_{p}\left(C_{*}\right) \rightarrow$ $H_{p}\left(C_{*}\right), C_{p} \rightarrow B_{p-1}\left(C_{*}\right)$, respectively. The Reidemeister torsion of $C_{*}$ with respect to bases $\left\{\mathbf{c}_{p}\right\}_{p=0}^{n},\left\{\mathbf{h}_{p}\right\}_{p=0}^{n}$ is the alternating product

$$
\mathrm{T}\left(C_{*},\left\{\mathbf{c}_{p}\right\}_{0}^{n},\left\{\mathbf{h}_{p}\right\}_{0}^{n}\right)=\prod_{p=0}^{n}\left[\mathbf{b}_{p} \oplus \ell_{p}\left(\mathbf{h}_{p}\right) \oplus s_{p}\left(\mathbf{b}_{p-1}\right), \mathbf{c}_{p}\right]^{(-1)^{(p+1)}},
$$

where $\left[\mathbf{e}_{p}, \mathbf{f}_{p}\right]$ denotes the determinant of the change-base-matrix from basis $\mathbf{f}_{p}$ to $\mathbf{e}_{p}$ of $C_{p}$.

Remark 1.2. Milnor proved that the Reidemeister torsion does not depend on bases $\mathbf{b}_{p}$, sections $s_{p}, \ell_{p}$ [17]. Let $\mathbf{c}_{p}^{\prime}, \mathbf{h}_{p}^{\prime}$ be other bases respectively for $C_{p}$, $H_{p}\left(C_{*}\right)$. Then, by an easy computation we have the following change-baseformula:

$$
\mathrm{T}\left(C_{*},\left\{\mathbf{c}_{p}^{\prime}\right\}_{0}^{n},\left\{\mathbf{h}_{p}^{\prime}\right\}_{0}^{n}\right)=\prod_{p=0}^{n}\left(\frac{\left[\mathbf{c}_{p}^{\prime}, \mathbf{c}_{p}\right]}{\left[\mathbf{h}_{p}^{\prime}, \mathbf{h}_{p}\right]}\right)^{(-1)^{p}} \mathrm{~T}\left(C_{*},\left\{\mathbf{c}_{p}\right\}_{0}^{n},\left\{\mathbf{h}_{p}\right\}_{0}^{n}\right) .
$$

By the independence of the Reidemeister torsion from $\mathbf{b}_{p}$ and sections $s_{p}, \ell_{p}$, formula (1.1) is easily obtained. Note that if, for example, $\left[\mathbf{c}_{p}^{\prime}, \mathbf{c}_{p}\right]=1$, $\left[\mathbf{h}_{p}^{\prime}, \mathbf{h}_{p}\right]=-1$, then the torsions are the same for odd $n$, and torsions have opposite sign for even $n$.

It follows from the Snake Lemma that a short-exact sequence of chain complexes

$$
0 \rightarrow A_{*} \stackrel{l}{\longrightarrow} B_{*} \stackrel{\pi}{\longrightarrow} D_{*} \rightarrow 0
$$


yields a long-exact sequence of vector spaces $C_{*}$ of length $3 n+2$. Namely,

$$
C_{*}: \cdots \rightarrow H_{p}\left(A_{*}\right) \stackrel{l_{p}}{\longrightarrow} H_{p}\left(B_{*}\right) \stackrel{\pi_{p}}{\longrightarrow} H_{p}\left(D_{*}\right) \stackrel{\delta_{p}}{\longrightarrow} H_{p-1}\left(A_{*}\right) \rightarrow \cdots,
$$

where $C_{3 p}=H_{p}\left(D_{*}\right), C_{3 p+1}=H_{p}\left(A_{*}\right)$, and $C_{3 p+2}=H_{p}\left(B_{*}\right)$.

Clearly, the bases $\mathbf{h}_{p}^{D}, \mathbf{h}_{p}^{A}$, and $\mathbf{h}_{p}^{B}$ serve as bases for $C_{3 p}, C_{3 p+1}$, and $C_{3 p+2}$, respectively.

The following result of Milnor states that the alternating product of the torsions of the chain complexes in (1.2) is equal to the torsion of (1.3). More precisely,

TheORem 1.3 ([17]). Let $\mathbf{c}_{p}^{A}, \mathbf{c}_{p}^{B}$, and $\mathbf{c}_{p}^{D}$ be bases respectively for $A_{p}$, $B_{p}$, and $D_{p}$. Let $\mathbf{h}_{p}^{A}, \mathbf{h}_{p}^{B}$, and $\mathbf{h}_{p}^{D}$ be bases of $H_{p}\left(A_{*}\right), H_{p}\left(B_{*}\right)$, and $H_{p}\left(D_{*}\right)$, respectively. If, moreover, $\mathbf{c}_{p}^{A}, \mathbf{c}_{p}^{B}, \mathbf{c}_{p}^{D}$ are compatible in the sense that $\left[\mathbf{c}_{p}^{B}, \mathbf{c}_{p}^{A} \oplus\right.$ $\left.\widetilde{\mathbf{c}_{p}^{D}}\right]= \pm 1$, where $\pi\left(\widetilde{\mathbf{c}_{p}^{D}}\right)=\mathbf{c}_{p}^{D}$, then

$$
\begin{aligned}
\mathrm{T}\left(B_{*},\left\{\mathbf{c}_{p}^{B}\right\}_{0}^{n},\left\{\mathbf{h}_{p}^{B}\right\}_{0}^{n}\right)= & \mathrm{T}\left(A_{*},\left\{\mathbf{c}_{p}^{A}\right\}_{0}^{n},\left\{\mathbf{h}_{p}^{A}\right\}_{0}^{n}\right) \mathrm{T}\left(D_{*},\left\{\mathbf{c}_{p}^{D}\right\}_{p=0}^{n},\left\{\mathbf{h}_{p}^{D}\right\}_{0}^{n}\right) \\
& \times \mathrm{T}\left(C_{*},\left\{\mathbf{c}_{3 p}\right\}_{0}^{3 n+2},\{0\}_{0}^{3 n+2}\right),
\end{aligned}
$$

where $\left[\mathbf{e}_{p}, \mathbf{f}_{p}\right]$ is the determinant of the change-base-matrix from basis $\mathbf{f}_{p}$ to $\mathbf{e}_{p}$ of $B_{p}$.

For future reference, let us prove the following sum-lemma:

Lemma 1.4. Let $A_{*}, D_{*}$ be two chain complexes. Let $\mathbf{c}_{p}^{A}, \mathbf{c}_{p}^{D}, \mathbf{h}_{p}^{A}$, and $\mathbf{h}_{p}^{D}$ be bases of $A_{p}, D_{p}, H_{p}\left(A_{*}\right)$, and $H_{p}\left(D_{*}\right)$, respectively. Then,

$$
\begin{aligned}
\mathrm{T}\left(A_{*} \oplus D_{*},\left\{\mathbf{c}_{p}^{A} \oplus \mathbf{c}_{p}^{D}\right\}_{0}^{n},\left\{\mathbf{h}_{p}^{A} \oplus \mathbf{h}_{p}^{D}\right\}_{0}^{n}\right) \\
=\mathrm{T}\left(A_{*},\left\{\mathbf{c}_{p}^{A}\right\}_{0}^{n},\left\{\mathbf{h}_{p}^{A}\right\}_{0}^{n}\right) \mathrm{T}\left(D_{*},\left\{\mathbf{c}_{p}^{D}\right\}_{0}^{n},\left\{\mathbf{h}_{p}^{D}\right\}_{0}^{n}\right) .
\end{aligned}
$$

Proof. Clearly, we have the following short exact sequence

$$
0 \rightarrow A_{*} \stackrel{\iota}{\longrightarrow} A_{*} \oplus D_{*} \stackrel{\pi}{\longrightarrow} D_{*} \rightarrow 0,
$$

where for $p=0, \ldots, n, \iota_{p}: A_{p} \rightarrow A_{p} \oplus D_{p}$ is the inclusion, and $\pi_{p}$ : $A_{p} \oplus D_{p} \rightarrow D_{p}$ is the projection.

Note also that the bases $\mathbf{c}_{p}^{A}, \mathbf{c}_{p}^{A} \oplus \mathbf{c}_{p}^{D}$, and $\mathbf{c}_{p}^{D}$ are compatible, where one can consider the inclusion as a section of $\pi_{p}: A_{p} \oplus D_{p} \rightarrow D_{p}$. Then, by Theorem 1.3, we obtain that $\mathrm{T}\left(A_{*} \oplus D_{*},\left\{\mathbf{c}_{p}^{A} \oplus \mathbf{c}_{p}^{D}\right\}_{0}^{n},\left\{\mathbf{h}_{p}^{A} \oplus \mathbf{h}_{p}^{D}\right\}_{0}^{n}\right)=$ $\mathbf{T}\left(A_{*},\left\{\mathbf{c}_{p}^{A}\right\}_{0}^{n},\left\{\mathbf{h}_{p}^{A}\right\}_{0}^{n}\right) \mathbf{T}\left(D_{*},\left\{\mathbf{c}_{p}^{D}\right\}_{0}^{n},\left\{\mathbf{h}_{p}^{D}\right\}_{0}^{n}\right) \mathbf{T}\left(C_{*},\left\{\mathbf{c}_{3 p}\right\}_{0}^{3 n+2},\{0\}_{0}^{3 n+2}\right)$, where $C_{*}$ is the long exact sequence obtained from short-exact sequence (1.4). Namely,

$$
C_{*}: 0 \rightarrow H_{n}\left(A_{*}\right) \stackrel{l_{n}}{\longrightarrow} H_{n}\left(A_{*} \oplus D_{*}\right) \stackrel{\pi_{n}}{\longrightarrow} H_{n}\left(D_{*}\right) \stackrel{\delta_{n}}{\longrightarrow} H_{n-1}\left(A_{*}\right) \rightarrow \cdots .
$$


Considering the projection $H_{p}\left(A_{*} \oplus D_{*}\right) \rightarrow H_{p}\left(A_{*}\right)$ as a section for $H_{p}\left(A_{*}\right) \rightarrow H_{p}\left(A_{*} \oplus D_{*}\right)$, the inclusion $H_{p}\left(D_{*}\right) \rightarrow H_{p}\left(A_{*} \oplus D_{*}\right)$ for $H_{p}\left(A_{*} \oplus D_{*}\right) \rightarrow H_{p}\left(D_{*}\right)$, and the zero map $H_{p-1}\left(A_{*}\right) \rightarrow H_{p}\left(D_{*}\right)$ for $H_{p}\left(D_{*}\right) \rightarrow H_{p-1}\left(A_{*}\right)$, we get $\mathrm{T}\left(C_{*}\right)=1$.

This completes the proof of Lemma 1.4.

Independently, it is explained in [1], [22] that a general chain complex can (unnaturally) be split as a direct sum of an acyclic and $\partial$-zero chain complexes. Moreover, it is proved independently in [1, Proposition 1.5] and [22, Theorem 2.0.4] that the Reidemeister torsion $\mathrm{T}\left(C_{*}\right)$ of a general complex $C_{*}$ can be interpreted as an element of $\bigotimes_{p=0}^{n}\left(\operatorname{det}\left(H_{p}\left(C_{*}\right)\right)\right)^{(-1)^{p+1}}$. For detailed proof and further information, we may refer the readers to [1], [22].

Definition 1.5. A symplectic chain complex of length $q$ is $\left(C_{*}, \partial_{*}\right.$, $\left.\left\{\omega_{*, q-*}\right\}\right)$, where

$$
C_{*}: 0 \rightarrow C_{q} \stackrel{\partial_{q}}{\longrightarrow} C_{q-1} \rightarrow \cdots \rightarrow C_{q / 2} \rightarrow \cdots \rightarrow C_{1} \stackrel{\partial_{1}}{\longrightarrow} C_{0} \rightarrow 0
$$

is a chain complex with $q \equiv 2(\bmod 4)$, and for $p=0, \ldots, q, \omega_{p, q-p}$ : $C_{p} \times C_{q-p} \rightarrow \mathrm{R}$ is a $\partial$-compatible anti-symmetric non-degenerate bilinear form. To be more precise,

$$
\begin{aligned}
\omega_{p, q-p}\left(\partial_{p+1} a, b\right) & =(-1)^{p+1} \omega_{p+1, q-(p+1)}\left(a, \partial_{q-p} b\right), \\
\omega_{p, q-p}(a, b) & =(-1)^{p(q-p)} \omega_{q-p, p}(b, a) .
\end{aligned}
$$

Note that by $q \equiv 2(\bmod 4)$, we easily have $\omega_{p, q-p}(a, b)=$ $(-1)^{p} \omega_{q-p, p}(b, a)$. It follows from the $\partial$-compatibility of the non-degenerate anti-symmetric bilinear maps $\omega_{p, q-p}: C_{p} \times C_{q-p} \rightarrow \mathrm{R}$ that one can easily extend these to homologies [22].

Definition 1.6. Let $C_{*}$ be a symplectic chain complex. We say that bases $\mathbf{c}_{p}$ of $C_{p}$ and $\mathbf{c}_{q-p}$ of $C_{q-p}$ are $\omega$-compatible if the matrix of $\omega_{p, q-p}$ in bases $\mathbf{c}_{p}, \mathbf{c}_{q-p}$ equals the $k \times k$ identity matrix $\mathrm{I}_{k \times k}$ when $p$ less than $\frac{q}{2}$ and

$$
\left[\begin{array}{cc}
0_{l \times l} & \mathrm{I}_{l \times l} \\
-\mathrm{I}_{l \times l} & 0_{l \times l}
\end{array}\right]
$$

when $p=q / 2$, where $k=\operatorname{dim} C_{p}=\operatorname{dim} C_{q-p}$ and $2 l=\operatorname{dim} C_{q / 2}$.

Similarly, considering $\left[\omega_{p, q-p}\right]: H_{p}\left(C_{*}\right) \times H_{q-p}\left(C_{*}\right) \rightarrow \mathrm{R}$, one can also define the [ $\omega$ ]-compatibility of bases $\mathbf{h}_{p}$ of $H_{p}\left(C_{*}\right)$ and $\mathbf{h}_{q-p}$ of $H_{q-p}\left(C_{*}\right)$.

The existence of $\omega$-compatible bases enabled us to prove in [22] that a symplectic chain complex $C_{*}$ can be split $\omega$-orthogonally as a direct sum of an 
exact and $\partial$-zero symplectic complexes. Moreover, we proved Theorem 1.7, which is one of the main results of [22]. Namely,

Theorem 1.7 ([22]). Let $C_{*}$ be a symplectic chain complex. For $p=$ $0, \ldots, q$, let $\mathbf{c}_{p}, \mathbf{h}_{p}$ be any bases of $C_{p}, H_{p}\left(C_{*}\right)$, respectively. Then, for the Reidemeister torsion of $C_{*}$ with respect to $\left\{\mathbf{c}_{p}\right\}_{0}^{q},\left\{\mathbf{h}_{p}\right\}_{0}^{q}$, the following formula

$$
\mathrm{T}\left(C_{*},\left\{\mathbf{c}_{p}\right\}_{0}^{q},\left\{\mathbf{h}_{p}\right\}_{0}^{q}\right)=\prod_{p=0}^{(q / 2)-1}\left(\operatorname{det}\left[\omega_{p, q-p}\right]\right)^{(-1)^{p}} \sqrt{\operatorname{det}\left[\omega_{q / 2, q / 2}\right]}(-1)^{q / 2}
$$

holds, where $\operatorname{det}\left[\omega_{p, q-p}\right]$ is the determinant of the matrix of the non-degenerate pairing $\left[\omega_{p, q-p}\right]: H_{p}\left(C_{*}\right) \times H_{q-p}\left(C_{*}\right) \rightarrow \mathrm{R}$ in bases $\mathbf{h}_{p}, \mathbf{h}_{q-p}$.

The proof and unexplained subjects can be found in [22]. For further applications of Theorem 1.7, we refer the reader to [23], [24].

\section{The Reidemeister Torsion of a Manifold}

Let $M$ be an $m$-manifold with a cell decomposition $K$. If $\mathbf{c}_{p}=\left\{c_{1}^{p}, \ldots, c_{n_{p}}^{p}\right\}$ is the geometric basis for the $p$-cells $C_{p}(K ; \mathrm{Z}), p=0, \ldots, m$, then one can associate to $M$ the following chain complex

$$
0 \rightarrow C_{m}(K) \stackrel{\partial_{m}}{\longrightarrow} C_{m-1}(K) \rightarrow \cdots \rightarrow C_{1}(K) \stackrel{\partial_{1}}{\longrightarrow} C_{0}(K) \rightarrow 0,
$$

where $Z$ is the set of integers and $\partial_{p}$ is the usual boundary operator.

Definition 2.1. Let $M$ be an $m$-manifold with a cell decomposition $K$. For $p=0, \ldots, m$, let $\mathbf{c}_{p}$ and $\mathbf{h}_{p}$ be bases of $C_{p}(K ; \mathbf{Z})$ and $H_{p}(M ; \mathbf{Z})$, respectively. $\mathrm{T}\left(C_{*}(K),\left\{\mathbf{c}_{p}\right\}_{0}^{m},\left\{\mathbf{h}_{p}\right\}_{0}^{m}\right)$ is called the Reidemeister torsion of $M$.

Using similar arguments introduced in [22, Lemma 2.0.5], one can prove:

Lemma 2.2. The Reidemeister torsion of $M$ is independent of cell decomposition.

Hence, the Reidemeister torsion $\mathrm{T}\left(C_{*}(K),\left\{\mathbf{c}_{p}\right\}_{0}^{m},\left\{\mathbf{h}_{p}\right\}_{0}^{m}\right)$ of $M$ is welldefined. Thus, we let $\mathrm{T}\left(M,\left\{\mathbf{h}_{p}\right\}_{0}^{m}\right)$ denote the Reidemeister torsion of $M$ in the bases $\mathbf{h}_{p}$ of $H_{p}(M), p=0, \ldots, m$.

By [1, Proposition 1.5] and [22, Theorem 2.0.4], one concludes that the Reidemeister torsion of $M$ is an element of the dual of 1-dimensional vector space $\bigotimes_{p=0}^{n}\left(\operatorname{det}\left(H_{p}(M)\right)^{(-1)^{p}}\right.$. 


\section{Proof of The Main Result}

In this section, we provide the proof of Theorem 0.1 . To alleviate the notation, let us introduce the following which is used throughout the paper. Let $Y$ be an oriented closed connected manifold of dimension $d$. For $p=0, \ldots, d$, let $\mathbf{h}_{p}^{Y}$ and $\mathbf{h}_{d-p}^{Y}$ be bases of $H_{p}(Y)$ and $H_{d-p}(Y)$, respectively. We denote the matrix of the intersection pairing $(\cdot, \cdot)_{p, d-p}: H_{p}(Y) \times H_{d-p}(Y) \rightarrow \mathrm{R}$ in the bases $\mathbf{h}_{p}^{Y}$ and $\mathbf{h}_{d-p}^{Y}$ by $H_{p, d-p}(Y)$. As convention, we let $H_{p, d-p}(Y)=1$ when $H_{p}(Y)=H_{d-p}(Y)=0$.

\subsection{Torsion of oriented closed connected $2 m$-manifold with $m$ odd, $\chi \equiv 0(\bmod 4)$}

This section will explain the symplectic chain complex associated to compact even dimensional manifolds. Moreover, we provide the proof of Theorem 0.1 for oriented closed connected $2 m$-manifolds with $m$ odd and Euler characteristic $\chi \equiv 0(\bmod 4)$. Namely,

THEOREM 3.1. Let $M$ be an oriented closed connected $2 m$-manifold with $m$ odd and $\chi(M) \equiv 0(\bmod 4)$. For $p=0, \ldots, 2 m$, let $\mathbf{h}_{p}$ be a basis of $H_{p}(M)$. Then,

$$
\left|\mathrm{T}\left(M,\left\{\mathbf{h}_{p}\right\}_{0}^{2 m}\right)\right|=\prod_{p=0}^{m-1}\left|\operatorname{det} H_{p, 2 m-p}(M)\right|^{(-1)^{p}} \sqrt{\operatorname{det} H_{m, m}(M)}(-1)^{m} .
$$

Proof. Let $K$ be a cell decomposition of $M$. Let $K^{\prime}$ be the corresponding dual cell decomposition of $M$ associated to $K$.

Recall that one can get the dual cell decomposition $K^{\prime}$ as follows. Let $K=\left\{\sigma_{\alpha}^{k}\right\}_{\alpha, k}$ and let $\left\{\tau_{\alpha}^{k}\right\}_{\alpha, k}$ denote the first barycentric subdivision of $K$. Then, for each vertex $\sigma_{\alpha}^{0} \in K$, associate the $2 m$-cell $\left(\sigma_{\alpha}^{0}\right)^{\prime}=\bigcup_{\sigma_{\alpha}^{0} \in \tau_{\beta}^{2 m}} \tau_{\beta}^{2 m}$ given as the union of all $2 m$-simplices $\tau_{\beta}^{2 m}$ in the subdivision with $\sigma_{\alpha}^{0}$ as a vertex. For each $k$-simplex in the cell decomposition $K$, let $\left(\sigma_{\alpha}^{k}\right)^{\prime}=\bigcap_{\sigma_{\beta}^{0} \in \sigma_{\alpha}^{k}}\left(\sigma_{\beta}^{0}\right)^{\prime}$ be the intersection of the $2 m$-cells $\left(\sigma_{\beta}^{0}\right)^{\prime}$ associated to the $k+1$ vertices of $\sigma_{\alpha}^{k}$.

This enables us to obtain the dual cell decomposition $K^{\prime}=\left\{\Delta_{\alpha}^{2 m-k}=\right.$ $\left.\left(\sigma_{\alpha}^{k}\right)^{\prime}\right\}$ of $M$ corresponding to $K$. Since $\Delta_{\alpha}^{2 m-k}=\left(\sigma_{\alpha}^{k}\right)^{\prime}$ and $\sigma_{\alpha}^{k}$ meet transversely, by giving an orientation on $\sigma_{\alpha}^{k}$, one can take the dual orientation on $\Delta_{\alpha}^{2 m-k}$ to be the one such that at $P \in \sigma_{\alpha}^{k} \cap\left(\sigma_{\alpha}^{k}\right)^{\prime}, l_{P}\left(\sigma_{\alpha}^{k},\left(\sigma_{\alpha}^{k}\right)^{\prime}\right)=1$, where $\iota_{P}$ denotes the intersection number index at $P$. 
Note that the intersection pairings $(\cdot, \cdot)_{k, 2 m-k}: C_{k}(K ; \mathrm{Z}) \times C_{2 m-k}\left(K^{\prime} ; \mathrm{Z}\right) \rightarrow$ R satisfy the following: for all $\alpha \in C_{k}(K ; \mathrm{Z}), \beta \in C_{2 m-k}\left(K^{\prime} ; \mathrm{Z}\right)$

(i) $(\alpha, \beta)_{k, 2 m-k}=(-1)^{k(2 m-k)}(\beta, \alpha)_{2 m-k, k}$,

(ii) $\left(\alpha, \partial_{2 m-k} \beta\right)_{(k+1), 2 m-(k+1)}=(-1)^{2 m-k+1}\left(\partial_{k+1} \alpha, \beta\right)_{k, 2 m-k}$,

where $\partial$ denotes the boundary operator.

From the similar property of the intersection number index (i) follows. Using $\partial_{2 m-k}\left(\Delta_{\alpha}^{2 m-k}\right)=(-1)^{2 m-k+1}\left(\partial_{k}\left(\alpha_{\alpha}^{k}\right)\right)^{\prime}$, (ii) is obtained, (see, e.g., [10, p. 55]).

Thus, the intersection pairings $(\cdot, \cdot)_{k, 2 m-k}$ are $\partial$-compatible anti-symmetric bilinear maps.

Let $D_{p}=C_{p}(K ; \mathbf{Z}) \oplus C_{p}\left(K^{\prime} ; \mathbf{Z}\right)$. By defining $(\cdot, \cdot)_{p, 2 m-p}$ as 0 on $C_{p}(K ; \mathrm{Z}) \times C_{2 m-p}(K ; \mathrm{Z})$, and $C_{p}\left(K^{\prime} ; \mathrm{Z}\right) \times C_{2 m-p}\left(K^{\prime} ; \mathrm{Z}\right)$, the chain-complex $0 \rightarrow D_{2 m} \rightarrow D_{2 m-1} \rightarrow \cdots \rightarrow D_{m} \rightarrow \cdots \rightarrow D_{1} \rightarrow D_{0} \rightarrow 0$ becomes a symplectic chain complex.

Clearly, the intersection pairings can be extended to homologies $(\cdot, \cdot)_{p, 2 m-p}: H_{p}(M) \times H_{2 m-p}(M) \rightarrow \mathrm{R}$.

It follows from Theorem 1.7 that

$$
\begin{aligned}
\mathrm{T}\left(D_{*},\left\{\mathbf{c}_{p} \oplus \mathbf{c}_{p}^{\prime}\right\}_{0}^{2 m},\left\{\mathbf{h}_{p} \oplus \mathbf{h}_{p}\right\}_{0}^{2 m}\right) \\
\quad=\prod_{p=0}^{m-1}\left(\operatorname{det}\left[\omega_{p, 2 m-p}\right]\right)^{(-1)^{p}} \sqrt{\operatorname{det}\left[\omega_{m, m}\right]}{ }^{(-1)^{m}},
\end{aligned}
$$

where $\operatorname{det}\left[\omega_{k, 2 m-k}\right]$ is the determinant of

$$
\left[\omega_{k, 2 m-k}\right]=\left[\begin{array}{cc}
0 & (\cdot, \cdot)_{k, 2 m-k} \\
(\cdot, \cdot)_{k, 2 m-k} & 0
\end{array}\right]: H_{k}\left(D_{*}\right) \times H_{2 m-k}\left(D_{*}\right) \rightarrow \mathbf{R}
$$

in the bases $\mathbf{h}_{k} \oplus \mathbf{h}_{k}, \mathbf{h}_{2 m-k} \oplus \mathbf{h}_{2 m-k}$, where $(\cdot, \cdot)_{k, 2 m-k}: H_{k}(M) \times$ $H_{2 m-k}(M) \rightarrow \mathrm{R}$ is the extension of the intersection pairing $(\cdot, \cdot)_{k, 2 m-k}$ : $C_{k}(K ; \mathrm{Z}) \times C_{2 m-k}\left(K^{\prime} ; \mathrm{Z}\right) \rightarrow \mathrm{R}$.

Note that for $p=0, \ldots, m$,

$$
\operatorname{det}\left[\omega_{p, 2 m-p}\right]=(-1)^{\operatorname{dim} H_{p}(M)} \operatorname{det} H_{p, 2 m-p}(M)^{2} .
$$

Note also that since $(\cdot, \cdot)_{m, m}: H_{m}(M) \times H_{m}(M) \rightarrow \mathrm{R}$ is non-degenerate anti-symmetric, the matrix $H_{m, m}(M)$ has positive determinant. 
Hence, combining equations (3.1) and (3.2), we have

$$
\begin{aligned}
\mathrm{T}\left(D_{*},\right. & \left.\left\{\mathbf{c}_{p} \oplus \mathbf{c}_{p}^{\prime}\right\}_{0}^{2 m},\left\{\mathbf{h}_{p} \oplus \mathbf{h}_{p}\right\}_{0}^{2 m}\right) \\
& =(-1)^{\chi(M) / 2}\left(\prod_{p=0}^{m-1} \operatorname{det} H_{p, 2 m-p}(M)^{(-1)^{p}}\right)^{2} \operatorname{det} H_{m, m}(M)^{(-1)^{m}},
\end{aligned}
$$

where $\chi(M)$ is the Euler characteristic of $M$.

For an oriented closed connected $2 m$-manifold $M$ with $m$ odd, $\chi(M)$ is even (see, e.g., [15, p. 164]). By the assumption $\chi(M) \equiv 0(\bmod 4),(3.3)$ becomes

$$
\begin{aligned}
\mathrm{T}\left(D_{*},\left\{\mathbf{c}_{p} \oplus \mathbf{c}_{p}^{\prime}\right\}_{0}^{2 m},\right. & \left.\left\{\mathbf{h}_{p} \oplus \mathbf{h}_{p}\right\}_{0}^{2 m}\right) \\
= & \left(\prod_{p=0}^{m-1} \operatorname{det} H_{p, 2 m-p}(M)^{(-1)^{p}}\right)^{2} \operatorname{det} H_{m, m}(M)^{(-1)^{m}} .
\end{aligned}
$$

Now, if we consider the inclusion $C_{p}(K ; \mathrm{Z}) \rightarrow D_{p}$ and the projection $D_{p} \rightarrow C_{p}\left(K^{\prime} ; \mathrm{Z}\right)$, then we obtain the following short-exact sequence of chain complexes $0 \rightarrow C_{*}(K ; \mathrm{Z}) \rightarrow D_{*} \rightarrow C_{*}\left(K^{\prime} ; \mathrm{Z}\right) \rightarrow 0$.

Let us take the inclusion $s_{p}: C_{p}\left(K^{\prime} ; \mathrm{Z}\right) \rightarrow D_{p}$ as a section of $D_{p} \rightarrow$ $C_{p}\left(K^{\prime} ; \mathrm{Z}\right)$. Then, the determinant of the change-base-matrix from $\mathbf{c}_{p} \oplus s_{p}\left(\mathbf{c}_{p}^{\prime}\right)$ to $\mathbf{c}_{p} \oplus \mathbf{c}_{p}^{\prime}$ is equal to 1 , and hence the bases $\mathbf{c}_{p}$ of $C_{p}(K ; Z), \mathbf{c}_{p} \oplus s_{p}\left(\mathbf{c}_{p}^{\prime}\right)$ of $D_{p}$, and $\mathbf{c}_{p}^{\prime}$ of $C_{p}\left(K^{\prime} ; \mathrm{Z}\right)$ are compatible.

Thus, by Lemma 1.4 and Lemma 2.2, we get

$$
\mathrm{T}\left(D_{*},\left\{\mathbf{c}_{p} \oplus \mathbf{c}_{p}^{\prime}\right\}_{0}^{2 m},\left\{\mathbf{h}_{p} \oplus \mathbf{h}_{p}\right\}_{0}^{2 m}\right)=\left(\mathrm{T}\left(M,\left\{\mathbf{h}_{p}\right\}_{p=0}^{2 m}\right)\right)^{2} .
$$

Combining (3.4) and (3.5), we conclude the proof of Theorem 3.1.

\subsection{The torsion of oriented closed connected $4 k$-manifold with $\chi$ even}

THEOREM 3.2. If $M$ is an oriented closed connected $2 m$-manifold with $m$ even and $\chi(M)$ even, and if $\mathbf{h}_{p}$ is a basis of $H_{p}(M), p=0, \ldots, 2 m$, then

$$
\left|\mathrm{T}\left(M,\left\{\mathbf{h}_{p}\right\}_{0}^{2 m}\right)\right|=\prod_{p=0}^{m-1}\left|\operatorname{det} H_{p, 2 m-p}(M)\right|^{(-1)^{p}}{\sqrt{\left|\operatorname{det} H_{m, m}(M)\right|}}^{(-1)^{m}} .
$$

Proof. Let us consider $N=M \times \mathrm{S}^{2}$, where $\mathrm{S}^{2}$ is the unit 2-sphere. Clearly, $N$ is an oriented closed connected $2(m+1)$-manifold with $m+1$ odd and $\chi(N)=0(\bmod 4)$. Let us also consider the usual CW structure of $\mathrm{s}^{2}$ with 
two cells, say, $\mathbf{c}_{0}^{\prime}, \mathbf{c}_{2}^{\prime}$. Let $\mathbf{h}_{0}^{\prime}$ and $\mathbf{h}_{2}^{\prime}$ be bases of homologies of $\mathrm{S}^{2}$ so that $\left(\mathbf{h}_{0}^{\prime}, \mathbf{h}_{2}^{\prime}\right)_{0,2}=1$.

For $p=2 m+1,2 m+2, C_{p}(N)=C_{p-2}(M) \otimes C_{2}\left(\mathrm{~S}^{2}\right)$, for $p=2, \ldots, 2 m$, $C_{p}(N)=C_{p-2}(M) \otimes C_{2}\left(\mathrm{~S}^{2}\right) \oplus C_{p}(M) \otimes C_{0}\left(\mathrm{~S}^{2}\right)$, and for $p=0,1, C_{p}(N)=$ $C_{p}(M) \otimes C_{0}\left(\mathrm{~S}^{2}\right)$.

Clearly, we have (3.6)

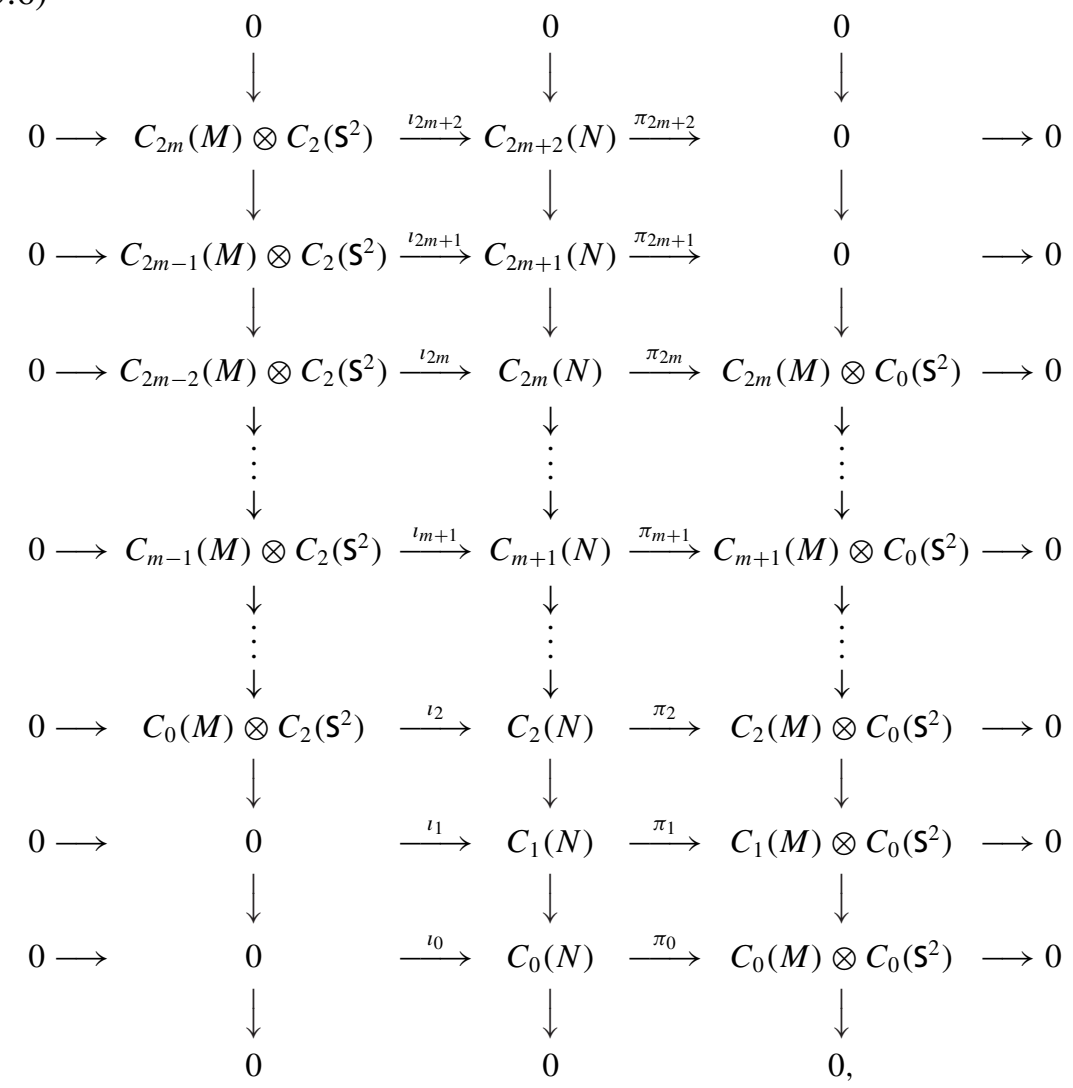

where $l_{p}$ is the inclusion, $\pi_{p}$ is the projection $p=0, \ldots, 2 m+2$.

Note that the bases of the chain complexes in (3.6) are compatible. $C_{p}(M) \otimes$ $C_{0}\left(\mathrm{~S}^{2}\right) \cong C_{p}(M)$ and $C_{p}(M) \otimes C_{2}\left(\mathrm{~S}^{2}\right) \cong C_{p}(M)$. From Lemma 1.4 it follows that $\mathrm{T}\left(C_{*}(N)\right)=\left(\mathrm{T}\left(C_{*}(M)\right)^{2}\right.$.

Using the Künneth formula (see, e.g., [11, p. 275]), we get for $p=0,1$, $\mathbf{h}_{p}^{N}=\mathbf{h}_{p} \otimes \mathbf{h}_{0}^{\prime}, \mathbf{h}_{2 m+2-p}^{N}=\mathbf{h}_{2 m-p} \otimes \mathbf{h}_{2}^{\prime}$, for $p=2, \ldots, m, \mathbf{h}_{p}^{N}=\mathbf{h}_{p-2} \otimes \mathbf{h}_{2}^{\prime} \oplus$ $\mathbf{h}_{p} \otimes \mathbf{h}_{0}^{\prime}, \mathbf{h}_{2 m+2-p}^{N}=\mathbf{h}_{2 m-p} \otimes \mathbf{h}_{2}^{\prime} \oplus \mathbf{h}_{2 m+2-p} \otimes \mathbf{h}_{0}^{\prime}$, and $\mathbf{h}_{m+1}^{N}=\mathbf{h}_{m-1} \otimes \mathbf{h}_{2}^{\prime} \oplus$ $\mathbf{h}_{m+1} \otimes \mathbf{h}_{0}^{\prime}$ are bases of $H_{p}(N), H_{2 m+2-p}(N)$, for $p=0,1, p=2, \ldots, m$, and $p=m+1$, respectively. 
It follows from Theorem 3.1 that

$$
\mathrm{T}\left(N,\left\{\mathbf{h}_{p}^{N}\right\}_{0}^{2 m+2}\right)=\prod_{p=0}^{m}\left|\operatorname{det} H_{p, 2 m+2-p}(N)\right|^{(-1)^{p}}{\sqrt{\operatorname{det} H_{m+1, m+1}(N)}}^{(-1)^{m+1}} .
$$

By an easy computation, we get for $p=0,1$,

$$
\left|\operatorname{det} H_{p, 2 m+2-p}(N)\right|=\left|\operatorname{det} H_{p, 2 m-p}(M)\right|,
$$

for $p=2, \ldots, m$,

$$
\begin{gathered}
\left|\operatorname{det} H_{p, 2 m+2-p}(N)\right|=\left|\operatorname{det} H_{p, 2 m-p}(M)\right|\left|\operatorname{det} H_{p-2,2 m+2-p}(M)\right|, \\
\sqrt{\operatorname{det} H_{m+1, m+1}(N)}=\left|\operatorname{det} H_{m-1, m+1}(M)\right| .
\end{gathered}
$$

From (3.7)-(3.10) it follows that

$$
\mathrm{T}\left(N,\left\{\mathbf{h}_{p}^{N}\right\}_{0}^{2 m+2}\right)=\prod_{p=0}^{m-1}\left|\operatorname{det} H_{p, 2 m-p}(M)^{(-1)^{p}}\right|^{2}\left|\operatorname{det} H_{m, m}(M)\right|^{(-1)^{m}}
$$

Thus, by (3.11) and the fact that $\mathrm{T}\left(C_{*}(N)\right)=\left(\mathrm{T}\left(C_{*}(M)\right)^{2}\right.$, we conclude the proof Theorem 3.2.

\subsection{The torsion of oriented closed connected $4 k$-manifold}

TheOREM 3.3. Let $M$ be an oriented closed connected $2 m$-manifold with $m$ even and for $p=0, \ldots, 2 m$, let $\mathbf{h}_{p}$ be a basis of $H_{p}(M)$. Then, for the Reidemeister torsion of $M$, the formula

$$
\left|\mathrm{T}\left(M,\left\{\mathbf{h}_{p}\right\}_{0}^{2 m}\right)\right|=\prod_{p=0}^{m-1}\left|\operatorname{det} H_{p, 2 m-p}(M)\right|^{(-1)^{p}}{\sqrt{\left|\operatorname{det} H_{m, m}(M)\right|}}^{(-1)^{m}}
$$

is valid.

Proof. Let $N$ denote $M \times \mathrm{S}^{2} \times \mathrm{S}^{2} . N$ is a closed oriented 2( $\left.m+2\right)$-manifold with $m+2$ even, and $\chi(N)=0(\bmod 4)$. Let us also consider the usual CW structure of $\mathrm{S}^{2}$ with two cells, say, $\mathbf{c}_{0}^{\prime}, \mathbf{c}_{2}^{\prime}$. Let $\mathbf{h}_{0}^{\prime}$ and $\mathbf{h}_{2}^{\prime}$ be bases for homologies of $\mathrm{S}^{2}$ so that $\left(\mathbf{h}_{0}^{\prime}, \mathbf{h}_{2}^{\prime}\right)_{0,2}=1$.

Clearly, for $p=2 m+3,2 m+4, C_{p}(N)=C_{p-4}(M) \otimes C_{4}\left(\mathrm{~S}^{2} \times \mathrm{S}^{2}\right)$, for $p=$ $2 m+1,2 m+2, C_{p}(N)=C_{p-4}(M) \otimes C_{4}\left(\mathrm{~S}^{2} \times \mathrm{S}^{2}\right) \oplus C_{p-2}(M) \otimes C_{2}\left(\mathrm{~S}^{2} \times \mathrm{S}^{2}\right)$, for $p=4, \ldots, 2 m, C_{p}(N)=C_{p-4}(M) \otimes C_{4}\left(\mathrm{~S}^{2} \times \mathrm{S}^{2}\right) \oplus C_{p-2}(M) \otimes C_{2}\left(\mathrm{~S}^{2} \times\right.$ 
$\left.\mathrm{S}^{2}\right) \oplus C_{p}(M) \otimes C_{0}\left(\mathrm{~S}^{2} \times \mathrm{S}^{2}\right)$, for $p=2,3, C_{p}(N)=C_{p-2}(M) \otimes C_{2}\left(\mathrm{~S}^{2} \times\right.$ $\left.\mathrm{S}^{2}\right) \oplus C_{p}(M) \otimes C_{0}\left(\mathrm{~S}^{2} \times \mathrm{S}^{2}\right)$, for $p=0,1, C_{p}(N)=C_{p}(M) \otimes C_{0}\left(\mathrm{~S}^{2} \times \mathrm{S}^{2}\right)$.

Note that $C_{p}(M) \otimes C_{4}\left(\mathrm{~S}^{2} \times \mathrm{S}^{2}\right) \cong C_{p}(M), C_{p}(M) \otimes C_{2}\left(\mathrm{~S}^{2} \times \mathrm{S}^{2}\right) \cong$ $C_{p}(M) \oplus C_{p}(M)$, and $C_{p}(M) \otimes C_{0}\left(\mathrm{~S}^{2} \times \mathrm{S}^{2}\right) \cong C_{p}(M)$. Using the compatibility of the bases, and Lemma 1.4, we have $\mathrm{T}\left(C_{*}(N)\right)=\left(\mathrm{T}\left(C_{*}(M)\right)^{4}\right.$.

From the Künneth formula it follows that for $p=2 m+3,2 m+4, \mathbf{h}_{p}^{N}=$ $\mathbf{h}_{p-4} \otimes\left(\mathbf{h}_{2}^{\prime} \otimes \mathbf{h}_{2}^{\prime}\right)$, for $p=2 m+1,2 m+2, \mathbf{h}_{p}^{N}=\mathbf{h}_{p-4} \otimes\left(\mathbf{h}_{2}^{\prime} \otimes \mathbf{h}_{2}^{\prime}\right) \oplus$ $\mathbf{h}_{p-2} \otimes\left(\mathbf{h}_{2}^{\prime} \otimes \mathbf{h}_{0}^{\prime} \oplus \mathbf{h}_{0}^{\prime} \otimes \mathbf{h}_{2}^{\prime}\right)$, for $p=4, \ldots, 2 m, \mathbf{h}_{p}^{N}=\mathbf{h}_{p-4} \otimes\left(\mathbf{h}_{2}^{\prime} \otimes\right.$ $\left.\mathbf{h}_{2}^{\prime}\right) \oplus \mathbf{h}_{p-2} \otimes\left(\mathbf{h}_{2}^{\prime} \otimes \mathbf{h}_{0}^{\prime} \oplus \mathbf{h}_{0}^{\prime} \otimes \mathbf{h}_{2}^{\prime}\right) \oplus \mathbf{h}_{p} \otimes\left(\mathbf{h}_{0}^{\prime} \otimes \mathbf{h}_{0}^{\prime}\right)$, for $p=2,3, \mathbf{h}_{p}^{N}=$ $\mathbf{h}_{p-2} \otimes\left(\mathbf{h}_{2}^{\prime} \otimes \mathbf{h}_{0}^{\prime} \oplus \mathbf{h}_{0}^{\prime} \otimes \mathbf{h}_{2}^{\prime}\right) \oplus \mathbf{h}_{p} \otimes\left(\mathbf{h}_{0}^{\prime} \otimes \mathbf{h}_{0}^{\prime}\right)$, and for $p=0,1, \mathbf{h}_{p}^{N}=\mathbf{h}_{p} \otimes\left(\mathbf{h}_{0}^{\prime} \otimes \mathbf{h}_{0}^{\prime}\right)$ are bases of $H_{p}(N)$, respectively for $p=2 m+3,2 m+4, p=2 m+1,2 m+2$, $p=4, \ldots, 2 m$, for $p=2,3$, and $p=0,1$.

By Theorem 3.2, we get

$$
\begin{aligned}
& \left|\mathrm{T}\left(N,\left\{\mathbf{h}_{p}^{N}\right\}_{0}^{2 m+4}\right)\right| \\
& \quad=\prod_{p=0}^{m+1}\left|\operatorname{det} H_{p, 2 m+4-p}(N)\right|^{(-1)^{p}} \sqrt{\left|\operatorname{det} H_{m+2, m+2}(N)\right|} .
\end{aligned}
$$

An easy computation results that for $p=0,1$,

$$
\left|\operatorname{det} H_{p, 2 m+4-p}(N)\right|=\left|\operatorname{det} H_{p, 2 m-p}(M)\right|,
$$

for $p=2,3$,

$$
\left|\operatorname{det} H_{p, 2 m+4-p}(N)\right|=\left|\operatorname{det} H_{p-2,2 m-p+2}(M)\right|^{2}\left|\operatorname{det} H_{p, 2 m-p}(M)\right|,
$$

for $p=4, \ldots, m$,

$$
\begin{aligned}
\left|\operatorname{det} H_{p, 2 m+4-p}(N)\right|=\left|\operatorname{det} H_{p, 2 m-p}(M)\right|\left|\operatorname{det} H_{p-2,2 m-p+2}(M)\right|^{2} \\
\times\left|\operatorname{det} H_{p-4,2 m-p+4}(M)\right|,
\end{aligned}
$$

Hence, (3.12)-(3.17), and the fact $\mathrm{T}\left(C_{*}(N)\right)=\left(\mathrm{T}\left(C_{*}(M)\right)^{4}\right.$ complete the proof of Theorem 3.3. 
3.4. Torsion of oriented closed connected $2 m$-manifold with $m$ odd, $\chi \equiv 2(\bmod 4)$

The Euler characteristic $\chi(M)$ of an oriented closed connected $2 m$-manifold $M$ with $m$ odd is even. In Theorem 3.1, we obtained a formula for the Reidemeister torsion of such $M$ with $\chi(M) \equiv 0(\bmod 4)$. In this section, we consider the case when $\chi(M) \equiv 2(\bmod 4)$.

THEOREM 3.4. Let $M$ be an oriented closed connected $2 m$-manifold with $m$ odd and $\chi(M) \equiv 2(\bmod 4)$. For $p=0, \ldots, 2 m$, let $\mathbf{h}_{p}$ be a basis of $H_{p}(M)$. Then,

$$
\left|\mathrm{T}\left(M,\left\{\mathbf{h}_{p}^{M}\right\}_{0}^{2 m}\right)\right|=\prod_{p=0}^{m-1}\left|\operatorname{det} H_{p, 2 m-p}(M)\right|^{(-1)^{p}}{\sqrt{\left|\operatorname{det} H_{m, m}(M)\right|}}^{(-1)^{m}} .
$$

Proof. Let us consider $N=M \times \mathrm{S}^{2}$, where we take the usual $\mathrm{CW}$ structure of $S^{2}$ with two cells, say, $\mathbf{c}_{0}^{\prime}, \mathbf{c}_{2}^{\prime}$. Let $\mathbf{h}_{0}^{\prime}$ and $\mathbf{h}_{2}^{\prime}$ be bases for homologies of $\mathbf{S}^{2}$ so that $\left(\mathbf{h}_{0}^{\prime}, \mathbf{h}_{2}^{\prime}\right)_{0,2}=1$.

$N$ is an oriented closed connected $2(m+1)$-manifold with $m+1$ even and $\chi(N) \equiv 0(\bmod 4)$. By Theorem 3.2, we get

$$
\begin{aligned}
& \left|\mathrm{T}\left(N,\left\{\mathbf{h}_{p}^{N}\right\}_{0}^{2 m+2}\right)\right| \\
& \quad=\prod_{p=0}^{m}\left|\operatorname{det} H_{p, 2 m+2-p}(N)\right|^{(-1)^{p}} \sqrt{\left|\operatorname{det} H_{m+1, m+1}(N)\right|} .
\end{aligned}
$$

For $p=2 m+1,2 m+2, C_{p}(N)=C_{p-2}(M) \otimes C_{2}\left(\mathrm{~S}^{2}\right)$, for $p=2, \ldots, 2 m$, $C_{p}(N)=C_{p-2}(M) \otimes C_{2}\left(\mathrm{~S}^{2}\right) \oplus C_{p}(M) \otimes C_{0}\left(\mathrm{~S}^{2}\right)$, and for $p=0,1, C_{p}(N)=$ $C_{p}(M) \otimes C_{0}\left(\mathrm{~S}^{2}\right)$.

We obtain a chain complex like (3.6). From the compatibility of the bases of the chain complexes, the fact $C_{p}(M) \otimes C_{0}\left(\mathrm{~S}^{2}\right) \cong C_{p}(M), C_{p}(M) \otimes C_{2}\left(\mathrm{~S}^{2}\right) \cong$ $C_{p}(M)$, and Lemma 1.4 it follows that $\mathrm{T}\left(C_{*}(N)\right)=\left(\mathrm{T}\left(C_{*}(M)\right)^{2}\right.$.

Using (3.8)-(3.10), (3.18) becomes

$$
\left|\mathrm{T}\left(N,\left\{\mathbf{h}_{p}^{N}\right\}_{0}^{2 m+2}\right)\right|=\prod_{p=0}^{m-1}\left|\operatorname{det} H_{p, 2 m-p}(M)^{(-1)^{p}}\right|^{2}\left|\operatorname{det} H_{m, m}(M)\right|^{(-1)^{m}}
$$

By (3.19) and the fact that $\mathrm{T}\left(C_{*}(N)\right)=\left(\mathrm{T}\left(C_{*}(M)\right)^{2}\right.$, we conclude the proof of Theorem 3.4.

Theorem 3.1, Theorem 3.3, and Theorem 3.4 terminate the proof of Theorem 0.1 . 
In the following section, we discuss the Reidemeister torsion of oriented closed connected odd dimensional manifolds.

\subsection{The torsion of oriented closed connected odd dimensional manifold}

TheOREM 3.5. Let $M$ be an oriented closed connected m-manifold with $m$ odd. Let $\mathbf{h}_{p}$ be a basis for $H_{p}(M), p=0, \ldots, m$. Then, $\left|\mathrm{T}\left(M,\left\{\mathbf{h}_{p}\right\}_{0}^{m}\right)\right|=1$.

Proof. Consider $N=M \times \mathrm{S}^{m}$, where $\mathrm{S}^{m}$ is the unit $m$-sphere. $N$ is an oriented closed connected $2 m$-manifold with $m$ odd. Clearly $\chi(N)=0$. Consider also the usual $\mathrm{CW}$ structure of $\mathrm{S}^{m}$ with two cells, say, $\mathbf{c}_{0}^{\prime}, \mathbf{c}_{m}^{\prime}$. Let $\mathbf{h}_{0}^{\prime}$ and $\mathbf{h}_{m}^{\prime}$ be bases for homologies of $S^{m}$ so that $\left(\mathbf{h}_{0}^{\prime}, \mathbf{h}_{m}^{\prime}\right)_{0, m}=1$.

Then, we get $C_{m}(N)=C_{m}(M) \otimes C_{0}\left(\mathrm{~S}^{m}\right) \oplus C_{0}(M) \otimes C_{m}\left(\mathrm{~S}^{m}\right)$, for $p=$ $0, \ldots, m-1, C_{p}(N)=C_{p}(M) \otimes C_{0}\left(\mathrm{~S}^{m}\right)$, and for $p=m+1, \ldots, 2 m$, $C_{p}(N)=C_{p-m}(M) \otimes C_{m}\left(\mathrm{~S}^{m}\right)$.

We also have

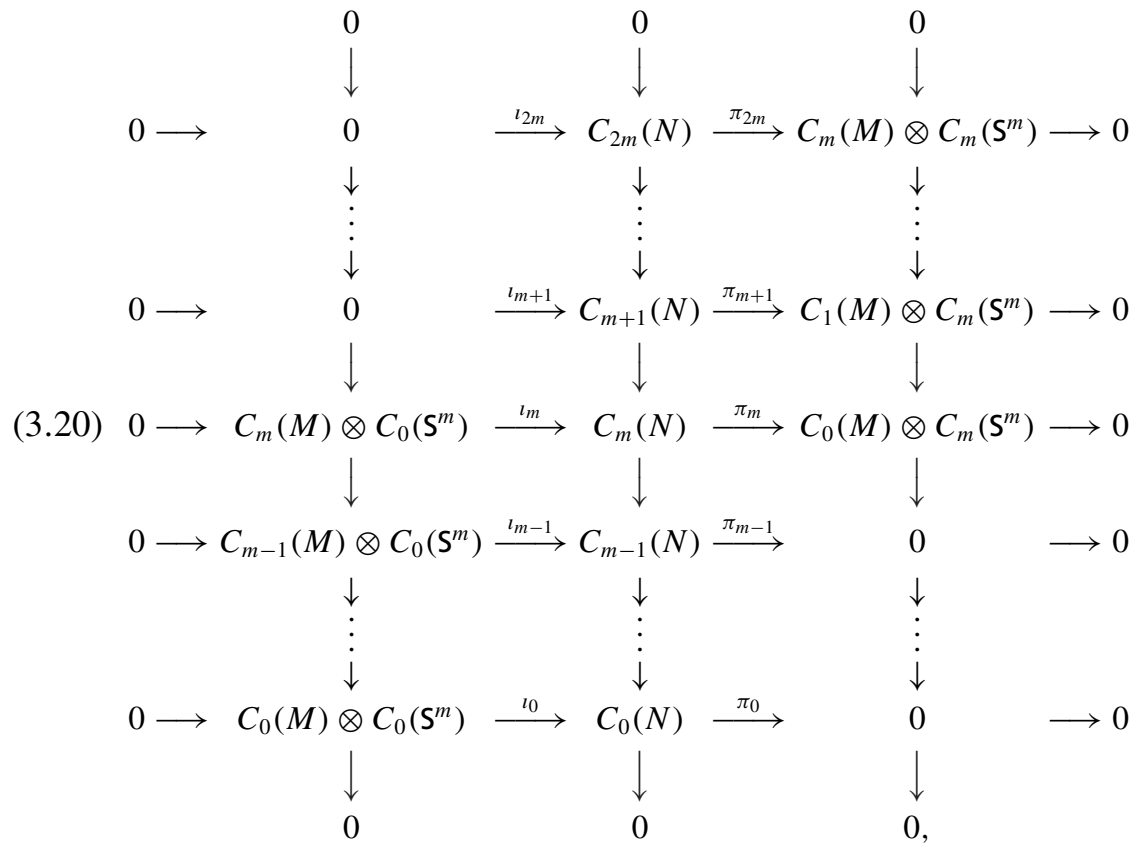

where $l_{p}$ is the inclusion, $\pi_{p}$ is the projection $p=0, \ldots, 2 m$.

Clearly, the bases of the chain complexes in (3.20) are compatible. Note also that $C_{p}(M) \otimes C_{m}\left(\mathrm{~S}^{m}\right) \cong C_{p}(M)$ and $C_{p}(M) \otimes C_{0}\left(\mathrm{~S}^{m}\right) \cong C_{p}(M)$. From Lemma 1.4 it follows that $\mathrm{T}\left(C_{*}(N)\right)=\left(\mathrm{T}\left(C_{*}(M)\right)^{2}\right.$.

By the Künneth formula, $\mathbf{h}_{p}^{N}=\mathbf{h}_{p} \otimes \mathbf{h}_{0}^{\prime}, \mathbf{h}_{2 m-p}^{N}=\mathbf{h}_{m-p} \otimes \mathbf{h}_{m}^{\prime}$, and $\mathbf{h}_{m}^{N}=$ $\mathbf{h}_{m} \otimes \mathbf{h}_{0}^{\prime} \oplus \mathbf{h}_{0} \otimes \mathbf{h}_{m}^{\prime}$ are bases of $H_{p}(N), H_{2 m-p}(N), p=0, \ldots, m$, respectively. 
It follows from Theorem 3.1 that

$$
\mathrm{T}\left(N,\left\{\mathbf{h}_{p}^{N}\right\}_{0}^{2 m}\right)=\prod_{p=0}^{m-1}\left|\operatorname{det} H_{p, 2 m-p}(N)\right|^{(-1)^{p}}{\sqrt{\operatorname{det} H_{m, m}(N)}}^{(-1)^{m}} .
$$

Note that

$$
\left|\operatorname{det} H_{0,2 m}(N)\right|=\left|\operatorname{det} H_{0, m}(M)\right| .
$$

For $p=1, \ldots, m-1$,

$$
\left|\operatorname{det} H_{p, 2 m-p}(N)\right|=\left|\operatorname{det} H_{m-p, m+p}(N)\right|=\left|\operatorname{det} H_{p, m-p}(M)\right| .
$$

Note also that since

$$
H_{m, m}(N)=\left[\begin{array}{cc}
0 & \left(h_{m}, h_{0}\right)_{m, 0} \\
-\left(h_{0}, h_{m}\right)_{0, m} & 0
\end{array}\right],
$$

we have

$$
\operatorname{det} H_{m, m}(N)=\operatorname{det} H_{0, m}(M)^{2} .
$$

Combining (3.21)-(3.24), and the fact that $\mathrm{T}\left(C_{*}(N)\right)=\left(\mathrm{T}\left(C_{*}(M)\right)^{2}\right.$, we get $\mathbf{T}\left(M,\left\{\mathbf{h}_{p}^{M}\right\}_{0}^{m}\right)^{2}=1$. This proves Theorem 3.5.

\section{Application}

In this section, we apply Theorem 0.1 to Riemann surfaces and Grassmannians.

\subsection{Compact Riemann surfaces}

Let $\Sigma_{g}$ be a compact oriented Riemann surface of genus $g \geq 1$ without boundary. Let $\Gamma=\left\{\Gamma_{1}, \ldots, \Gamma_{g}, \Gamma_{1+g}, \ldots, \Gamma_{2 g}\right\}$ be a canonical basis for $H_{1}\left(\Sigma_{g}\right)$, i.e., $\Gamma_{i}$ intersects $\Gamma_{i+g}$ once positively and does not intersect others. Then, we have

TheOREM 4.1. Let $\mathbf{h}_{0}, \mathbf{h}_{1}=\left\{\Omega_{i}\right\}_{1}^{2 g}$, and $\mathbf{h}_{2}$ be bases of $H_{0}\left(\Sigma_{g}\right), H_{1}\left(\Sigma_{g}\right)$, and $\mathrm{H}_{2}\left(\Sigma_{g}\right)$, respectively. Then,

$$
\left|\mathrm{T}\left(\Sigma_{g},\left\{\mathbf{h}_{p}\right\}_{0}^{2}\right)\right|=\left|\frac{\operatorname{det} H_{0,2}\left(\Sigma_{g}\right)}{\operatorname{det} \wp\left(\mathfrak{h}^{1}, \Gamma\right)}\right|,
$$

where $\mathbf{h}^{1}=\left\{\omega_{i}\right\}_{1}^{2 g}$ is the Poincare dual basis of $H^{1}\left(\Sigma_{g}\right)$ corresponding to the basis $\mathbf{h}_{1}$ of $H_{1}\left(\Sigma_{g}\right)$, where $\wp\left(\mathbf{h}^{1}, \Gamma\right)=\left[\int_{\Gamma_{i}} \omega_{j}\right]$ is the period matrix of $\mathbf{h}^{1}$ with respect to the canonical basis $\Gamma=\left\{\Gamma_{i}\right\}_{i=1}^{2 g}$ of $H_{1}\left(\Sigma_{g}\right)$. 
Proof. From Theorem 0.1 it follows that $\left|\mathrm{T}\left(\Sigma_{g},\left\{\mathbf{h}_{p}\right\}_{0}^{2}\right)\right|=\frac{\left|\operatorname{det} H_{0,2}\left(\Sigma_{g}\right)\right|}{\sqrt{\operatorname{det} H_{1,1}\left(\Sigma_{g}\right)}}$. For $\mathbf{h}_{1}=\left\{\Omega_{j}\right\}_{j=1}^{2 g}$, the non-degenerate skew-symmetric $2 g \times 2 g$-square matrix $H_{1,1}\left(\Sigma_{g}\right)$ is $\left[\Omega_{i j}\right]$, where $\Omega_{i j}=\left(\Omega_{i}, \Omega_{j}\right)_{1,1}$. By Poincaré duality, we also have $\Omega_{i j}=\int_{\Sigma_{g}} \omega_{i} \wedge \omega_{j}$. Change-base-formula results that $\sqrt{\operatorname{det}\left(H_{1,1}\left(\Sigma_{g}\right)\right)}=$ $\left|\operatorname{det}\left[\left(\Omega_{j}, \Gamma_{i}\right)_{1,1}\right]\right|$. If, moreover, we let $\gamma_{i} \in H^{1}\left(\Sigma_{g}\right)$ denote the Poincaré dual of $\Gamma_{i} \in H_{1}\left(\Sigma_{g}\right)$, then we have $\left(\Omega_{i}, \Gamma_{j}\right)_{1,1}=\int_{\Sigma_{g}} \omega_{i} \wedge \gamma_{j}=\int_{\Gamma_{i}} \omega_{j}$.

This completes the proof of Theorem 4.1.

Before ending this section, we also would like to apply Theorem 0.1 to $M \times N$, where $M=\Sigma_{g}, N=\Sigma_{g^{\prime}}$ are compact oriented Riemann surfaces of genus $g, g^{\prime} \geq 1$ without boundary.

Let us start with the following well-known properties of tensor (or Kronecker) product of square matrices. Recall that if $A=\left[a_{i j}\right]$ is an $m \times m$ and $B=\left[b_{i j}\right]$ is an $n \times n$ matrix with real entries, then the tensor product of $A$ and $B$ is the $m n \times m n$ block matrix $A \otimes B=\left[a_{i j} B\right]$, where $a_{i j} B$ is the $n \times n$ matrix obtained by multiplying the matrix $B$ with the scalar $a_{i j}$.

Recall that if $A, B, C, D$ are square matrices such that the products $A C$ and $B D$ exist, then $(A \otimes B)(C \otimes D)$ exists and $(A \otimes B)(C \otimes D)=(A C) \otimes(B D)$ (see, e.g., [21, p. 350]). Let $A$ be an $n \times n$ and $B$ be an $m \times m$ invertible matrix. Then, we clearly have $(A \otimes B)\left(A^{-1} \otimes B^{-1}\right)=I_{m \times m} \otimes I_{n \times n}$, where $I_{d \times d}$ is the $d \times d$ identity matrix. Note also that for the square matrices $A$ and $B$, we have $(A \otimes B)^{T}=A^{T} \otimes B^{T}$, where $A^{T}$ is the transpose of $A$. Finally, it is known that $\operatorname{det}(A \otimes B)=\operatorname{det}(A)^{n} \operatorname{det}(B)^{m}$; however, for the sake of completeness, we provide a proof for our case. More precisely,

Lemma 4.2. Let $A=\left[a_{i j}\right]$ be $2 g \times 2 g$ and $B=\left[b_{i j}\right]$ be a $2 g^{\prime} \times 2 g^{\prime}$ symmetric or skew-symmetric matrices with real entries. Then, $\operatorname{det}(A \otimes B)=$ $\operatorname{det}(A)^{2 g^{\prime}} \operatorname{det}(B)^{2 g}$.

Proof. By the spectral theorem of normal matrices, symmetric and skewsymmetric matrices are orthogonally diagonalizable. Thus, there exist orthogonal $2 g \times 2 g$ real matrix $P$ and $2 g^{\prime} \times 2 g^{\prime}$ real matrix $Q$ so that $P A P^{-1}=$ $D_{1}=\operatorname{diag}\left(\lambda_{1}, \ldots, \lambda_{2 g}\right), Q B Q^{-1}=D_{2}=\operatorname{diag}\left(\mu_{1} \ldots, \mu_{2 g^{\prime}}\right)$, respectively, where $\lambda_{1}, \ldots, \lambda_{2 g}$ and $\mu_{1}, \ldots, \mu_{2 g^{\prime}}$ are real. Then, we have $A \otimes B=$ $\left(P D_{1} P^{-1}\right) \otimes\left(Q D_{2} Q^{-1}\right)=(P \otimes Q)\left(D_{1} \otimes D_{2}\right)(P \otimes Q)^{-1}$.

Hence, $\operatorname{det}(A \otimes B)=\operatorname{det}\left(D_{1} \otimes D_{2}\right)=\operatorname{det}\left(D_{1}\right)^{2 g^{\prime}} \operatorname{det}\left(D_{2}\right)^{2 g}=$ $\operatorname{det}(A)^{2 g^{\prime}} \operatorname{det}(B)^{2 g}$.

This is the end of the proof of Lemma 4.2.

Corollary 4.3. Let $M=\Sigma_{g}$ and $N=\Sigma_{g^{\prime}}$ be closed oriented Riemann surfaces of genus $g, g^{\prime} \geq 1$, respectively. For $p=0,1,2$, let $\mathbf{h}_{p}$ and $\mathbf{h}_{p}^{\prime}$ be 
bases of $H_{p}(M)$ and $H_{p}(N)$, respectively. Then,

(4.1) $\left|\mathrm{T}\left(M \times N,\left\{\bigoplus_{i+j=p} \mathbf{h}_{i} \otimes \mathbf{h}_{j}^{\prime}\right\}_{p=0}^{4}\right)\right|=\left|\mathrm{T}\left(M,\left\{\mathbf{h}_{p}\right\}_{0}^{2}\right)\right|^{\chi(N)}\left|\mathrm{T}\left(N,\left\{\mathbf{h}_{p}^{\prime}\right\}_{0}^{2}\right)\right|^{\chi(M)}$.

Proof. From the Künneth formula it follows that $\mathbf{h}_{0} \otimes \mathbf{h}_{0}^{\prime}, \mathbf{h}_{1} \otimes \mathbf{h}_{0}^{\prime} \oplus \mathbf{h}_{0} \otimes \mathbf{h}_{1}^{\prime}$, $\mathbf{h}_{0} \otimes \mathbf{h}_{2}^{\prime} \oplus \mathbf{h}_{1} \otimes \mathbf{h}_{1}^{\prime} \oplus \mathbf{h}_{2} \otimes \mathbf{h}_{0}^{\prime}, \mathbf{h}_{1} \otimes \mathbf{h}_{2}^{\prime} \oplus \mathbf{h}_{2} \otimes \mathbf{h}_{1}^{\prime}$, and $\mathbf{h}_{2} \otimes \mathbf{h}_{2}^{\prime}$ are bases of $H_{0}(M \times N), H_{1}(M \times N), H_{2}(M \times N), H_{3}(M \times N)$, and $H_{4}(M \times N)$, respectively.

Using Theorem 0.1 , we obtain

$$
\begin{aligned}
& \left|\mathrm{T}\left(M \times N,\left\{\bigoplus_{i+j=p} \mathbf{h}_{i} \otimes \mathbf{h}_{j}^{\prime}\right\}_{p=0}^{4}\right)\right| \\
& \quad=\left|\operatorname{det} H_{0,4}(M \times N)\right|\left|\operatorname{det} H_{1,3}(M \times N)\right|^{-1} \sqrt{\left|\operatorname{det} H_{2,2}(M \times N)\right|} .
\end{aligned}
$$

It follows from Lemma 4.2 that

$$
\left|\operatorname{det} H_{0,4}(M \times N)\right|=\left|\operatorname{det} H_{0,2}(M)\right|\left|\operatorname{det} H_{0,2}(N)\right|
$$

$$
\begin{aligned}
\left|\operatorname{det} H_{1,3}(M \times N)\right|=\left|\operatorname{det} H_{0,2}(M)\right|^{\operatorname{dim} H_{1}(N)}\left|\operatorname{det} H_{1,1}(M)\right| \\
\times\left|\operatorname{det} H_{0,2}(N)\right|^{\operatorname{dim} H_{1}(M)}\left|\operatorname{det} H_{1,1}(N)\right|
\end{aligned}
$$

(4.5) $\left|\operatorname{det} H_{2,2}(M \times N)\right|$

$$
=\left|\operatorname{det} H_{0,2}(M)\right|^{2}\left|\operatorname{det} H_{0,2}(N)\right|^{2}\left|\operatorname{det} H_{1 \otimes 1}(M \times N)\right|,
$$

where $H_{1 \otimes 1}(M \times N)=\left[(\cdot, \cdot)\right.$ in $\left.\mathbf{h}_{1} \otimes \mathbf{h}_{1}^{\prime}\right]$.

By Lemma 4.2, we get

(4.6) $\operatorname{det} H_{1 \otimes 1}(M \times N)=\left(\operatorname{det} H_{1,1}(M)\right)^{\operatorname{dim} H_{1}(N)}\left(\operatorname{det} H_{1,1}(N)\right)^{\operatorname{dim} H_{1}(M)}$.

From (4.3)-(4.6) it follows that (4.2) is equal to

$$
\begin{aligned}
& \mid \mathrm{T}(M\left.\times N,\left\{\bigoplus_{i+j=p} \mathbf{h}_{i} \otimes \mathbf{h}_{j}^{\prime}\right\}_{p=0}^{4}\right) \mid \\
&=\left|\operatorname{det} H_{0,2}(M)\right|^{\chi(N)}\left|\operatorname{det} H_{1,1}(M)\right|^{-\chi(N) / 2} \\
& \times\left|\operatorname{det} H_{0,2}(N)\right|^{\chi(M)}\left|\operatorname{det} H_{1,1}(N)\right|^{-\chi(M) / 2} \\
&=\left|\mathrm{T}\left(M,\left\{\mathbf{h}_{p}\right\}_{p=0}^{2}\right)\right|^{\chi(N)}\left|\mathrm{T}\left(N,\left\{\mathbf{h}_{p}^{\prime}\right\}_{p=0}^{2}\right)\right|^{\chi(M)}
\end{aligned}
$$


This concludes the proof of Corollary 4.3.

Next, let us compute the Reidemeister torsion of the cartesian product $\times_{i=1}^{n} \Sigma_{g_{i}}$ of closed Riemann surfaces $\Sigma_{g_{1}}, \ldots, \Sigma_{g_{n}}$ of genus $g_{1}, \ldots, g_{n} \geq 1$, respectively. To do that, we shall first prove that formula (4.1) is valid for $M \times N$, where $M$ is an oriented closed connected $2 n$-manifold with $n \geq 1$ and $N=\Sigma_{g^{\prime}}$ is a closed oriented Riemann surfaces of genus $g^{\prime} \geq 1$. Namely,

COROLlary 4.4. Let $M$ be an oriented closed connected $2 n$-manifold with $n \geq 1$ and $N=\Sigma_{g^{\prime}}$ be a closed oriented Riemann surface of genus $g^{\prime} \geq 1$. For $i=0, \ldots, 2 n$, let $\mathbf{h}_{i}$ be a basis of $H_{i}(M)$. Let $\mathbf{h}_{j}^{\prime}$ be a basis of $H_{j}(N)$, $j=0,1,2$. Then,

$$
\begin{aligned}
\left|\mathrm{T}\left(M \times N,\left\{\bigoplus_{i+j=p} \mathbf{h}_{i} \otimes \mathbf{h}_{j}^{\prime}\right\}_{p=0}^{2 n+2}\right)\right| \\
\quad=\left|\mathrm{T}\left(M,\left\{\mathbf{h}_{p}\right\}_{0}^{2 n}\right)\right|^{\chi(N)}\left|\mathrm{T}\left(N,\left\{\mathbf{h}_{p}^{\prime}\right\}_{0}^{2}\right)\right|^{\chi(M)} .
\end{aligned}
$$

Proof. Using the Künneth formula, we get $\mathbf{h}_{0} \otimes \mathbf{h}_{0}^{\prime}, \mathbf{h}_{2 n} \otimes \mathbf{h}_{2}^{\prime}, \mathbf{h}_{1} \otimes \mathbf{h}_{0}^{\prime} \oplus$ $\mathbf{h}_{0} \otimes \mathbf{h}_{1}^{\prime}, \mathbf{h}_{2 n-1} \otimes \mathbf{h}_{2}^{\prime} \oplus \mathbf{h}_{2 n} \otimes \mathbf{h}_{1}^{\prime}$, and for $p=2, \ldots, n+1, \mathbf{h}_{p} \otimes \mathbf{h}_{0}^{\prime} \oplus$ $\mathbf{h}_{p-1} \otimes \mathbf{h}_{1}^{\prime} \oplus \mathbf{h}_{p-2} \otimes \mathbf{h}_{2}^{\prime}, \mathbf{h}_{2 n-p} \otimes \mathbf{h}_{2}^{\prime} \oplus \mathbf{h}_{2 n-p+1} \otimes \mathbf{h}_{1}^{\prime} \oplus \mathbf{h}_{2 n-p+2} \otimes \mathbf{h}_{0}^{\prime}$ are bases of $H_{0}(M \times N), H_{2 n+2}(M \times N), H_{1}(M \times N), H_{2 n+1}(M \times N)$, and for $p=2, \ldots, n+1, H_{p}(M \times N), H_{2 n+2-p}(M \times N)$, respectively.

It follows from Theorem 0.1 that

$$
\begin{aligned}
& \left|\mathrm{T}\left(M \times N,\left\{\bigoplus_{i+j=p} \mathbf{h}_{i} \otimes \mathbf{h}_{j}^{\prime}\right\}_{p=0}^{2 n+2}\right)\right| \\
& =\prod_{p=0}^{n}\left|\operatorname{det} H_{p, 2 n+2-p}(M \times N)\right|^{(-1)^{p}}{\sqrt{\left|\operatorname{det} H_{p, 2 n+2-p}(M \times N)\right|}}^{(-1)^{n+1}} .
\end{aligned}
$$

Using Lemma 4.2, we get

$$
\begin{aligned}
& \text { (4.9) } \begin{array}{r}
\left|\operatorname{det} H_{0,2 n+2}(M \times N)\right| \\
=\left|\operatorname{det} H_{0,2 n}(M)\right|^{\operatorname{dim} H_{0}(N)}\left|\operatorname{det} H_{0,2}(N)\right|^{\operatorname{dim} H_{0}(M)}, \\
\text { (4.10) }\left|\operatorname{det} H_{1,2 n+1}(M \times N)\right| \\
=\left|\operatorname{det} H_{1,2 n-1}(M)\right|^{\operatorname{dim} H_{0}(N)}\left|\operatorname{det} H_{0,2}(N)\right|^{\operatorname{dim} H_{1}(M)} \\
\times\left|\operatorname{det} H_{0,2 n}(M)\right|^{\operatorname{dim} H_{1}(N)}\left|\operatorname{det} H_{1,1}(N)\right|^{\operatorname{dim} H_{0}(M)}
\end{array}
\end{aligned}
$$


for $p=2, \ldots, n$,

(4.11) $\left|\operatorname{det} H_{p, 2 n+2-p}(M \times N)\right|=\left|\operatorname{det} H_{p, 2 n-p}(M)\right|^{\operatorname{dim} H_{0}(N)}$

$\times\left|\operatorname{det} H_{p-1,2 n-p+1}(M)\right|^{\operatorname{dim} H_{1}(N)}\left|\operatorname{det} H_{p-2,2 n-p+2}(M)\right|^{\operatorname{dim} H_{0}(N)}$

$\times\left|\operatorname{det} H_{0,2}(N)\right|^{\operatorname{dim} H_{p}(M)+\operatorname{dim} H_{p-2}(M)}\left|\operatorname{det} H_{1,1}(N)\right|^{\operatorname{dim} H_{p-1}(M)}$,

(4.12) $\sqrt{\left|\operatorname{det} H_{n+1, n+1}(M \times N)\right|}=\left|\operatorname{det} H_{n-1, n+1}(M)\right|^{\operatorname{dim} H_{0}(N)}$

$$
\begin{aligned}
\times\left|\operatorname{det} H_{0,2}(N)\right|^{\operatorname{dim} H_{n-1}(M)}\left|\operatorname{det} H_{n, n}(M)\right|^{\operatorname{dim} H_{1}(N) / 2} \\
\times\left|\operatorname{det} H_{1,1}(N)\right|^{\operatorname{dim} H_{n}(M) / 2} .
\end{aligned}
$$

Using (4.9)-(4.12), (4.8) is equal to

$$
\begin{aligned}
& \prod_{p=2}^{n}\left\{\left|\operatorname{det} H_{p, 2 n-p}(M)\right|^{\operatorname{dim} H_{0}(N)}\left|\operatorname{det} H_{p-1,2 n-p+1}(M)\right|^{\operatorname{dim} H_{1}(N)}\right. \\
& \left.\quad \times\left|\operatorname{det} H_{p-2,2 n-p+2}(M)\right|^{\operatorname{dim} H_{0}(N)}\right\}^{(-1)^{p}} \prod_{p=2}^{n}\left\{\left|\operatorname{det} H_{0,2}(N)\right|^{\operatorname{dim} H_{p}(M)}\right. \\
& \left.\quad \times\left|\operatorname{det} H_{1,1}(N)\right|^{\operatorname{dim} H_{p-1}(M)}\left|\operatorname{det} H_{0,2}(N)\right|^{\operatorname{dim} H_{p-2}(M)}\right\}^{(-1)^{p}} \\
& \quad \times\left|\operatorname{det} H_{0,2 n}(M)\right|^{\operatorname{dim} H_{0}(N)}\left|\operatorname{det} H_{0,2}(N)\right|^{\operatorname{dim} H_{0}(M)} \\
& \quad \times\left|\operatorname{det} H_{0,2 n}(M)\right|^{-\operatorname{dim} H_{1}(N)}\left|\operatorname{det} H_{1,1}(N)\right|^{-\operatorname{dim} H_{0}(M)} \\
& \quad \times\left|\operatorname{det} H_{1,2 n-1}(M)\right|^{-\operatorname{dim} H_{0}(N)}\left|\operatorname{det} H_{0,2}(N)\right|^{-\operatorname{dim} H_{1}(M)} \\
& \quad \times\left\{\left|\operatorname{det} H_{n-1, n+1}(M)\right|^{\operatorname{dim} H_{0}(N)}\left|\operatorname{det} H_{0,2}(N)\right|^{\operatorname{dim} H_{n-1}(M)}\right. \\
& \left.\quad \times\left|\operatorname{det} H_{n, n}(M)\right|^{\operatorname{dim} H_{1}(N) / 2}\left|\operatorname{det} H_{1,1}(N)\right|^{\operatorname{dim} H_{n}(M) / 2}\right\}^{(-1)^{n+1}} .
\end{aligned}
$$

An easy computation gives us

$$
\begin{aligned}
& \prod_{p=2}^{n}\left\{\left|\operatorname{det} H_{p, 2 n-p}(M)\right|\left|\operatorname{det} H_{p-1,2 n-p+1}(M)\right|^{\operatorname{dim} H_{1}(N)}\right. \\
& \left.\quad \times\left|\operatorname{det} H_{p-2,2 n-p+2}(M)\right|\right\}^{(-1)^{p}}\left|\operatorname{det} H_{0,2 n}(M)\right|^{\operatorname{dim} H_{0}(N)-\operatorname{dim} H_{1}(N)} \\
& \quad \times\left|\operatorname{det} H_{1,2 n-1}(M)\right|^{-\operatorname{dim} H_{0}(N)}\left|\operatorname{det} H_{n-1, n+1}(M)\right|^{(-1)^{n+1} \operatorname{dim} H_{0}(N)}
\end{aligned}
$$




$$
\begin{aligned}
& \times\left|\operatorname{det} H_{n, n}(M)\right|^{(-1)^{n+1} \operatorname{dim} H_{1}(N) / 2} \\
= & \left(\prod_{p=0}^{n-1}\left|\operatorname{det} H_{p, 2 n-p}(M)\right|^{(-1)^{p}}\right)^{\chi(N)}\left(\left|\operatorname{det} H_{n, n}(M)\right|^{\left((-1)^{n+1}\right) / 2}\right)^{\chi(N)} \\
= & \left|\mathrm{T}\left(M,\left\{\mathbf{h}_{p}\right\}_{p=0}^{2 n}\right)\right|^{\chi(N)} .
\end{aligned}
$$

Clearly, we have

$$
\begin{aligned}
\prod_{p=2}^{n}\{ & \left.\left|\operatorname{det} H_{0,2}(N)\right|^{\operatorname{dim} H_{p}(M)+\operatorname{dim} H_{p-2}(M)}\left|\operatorname{det} H_{1,1}(N)\right|^{\operatorname{dim} H_{p-1}(M)}\right\}^{(-1)^{p}} \\
& \times\left|\operatorname{det} H_{0,2}(N)\right|^{\operatorname{dim} H_{0}(M)-\operatorname{dim} H_{1}(M)}\left|\operatorname{det} H_{1,1}(N)\right|^{-\operatorname{dim} H_{0}(M)} \\
& \times\left|\operatorname{det} H_{0,2}(N)\right|^{(-1)^{n+1} \operatorname{dim} H_{n+1}(M) / 2}\left|\operatorname{det} H_{0,2}(N)\right|^{(-1)^{n+1} \operatorname{dim} H_{n-1}(M) / 2} \\
& \times\left|\operatorname{det} H_{1,1}(N)\right|^{(-1)^{n+1} \operatorname{dim} H_{n}(M) / 2} \\
= & \left|\operatorname{det} H_{0,2}(N)\right|^{\chi(M)}\left|\operatorname{det} H_{1,1}(N)\right|^{-\chi(M) / 2}=\left|\mathrm{T}\left(N,\left\{\mathbf{h}_{p}^{\prime}\right\}_{p=0}^{2}\right)\right|^{\chi(M)}
\end{aligned}
$$

Combining (4.14) and (4.15), we obtain (4.7).

This finishes the proof of Corollary 4.4 .

In particular, considering the cartesian product of closed oriented Riemann surfaces of genus $\geq 1$ and applying Corollary 4.4, we have

COROLlaRY 4.5. Let $\Sigma_{g_{1}}, \ldots, \Sigma_{g_{n}}$ be closed oriented Riemann surfaces of genus $g_{1}, \ldots, g_{n} \geq 1$, respectively. For $p=0,1,2$, and $i=1, \ldots, n$, let $\mathbf{h}_{p, i}$ be a basis of $H_{p}\left(\Sigma_{g_{i}}\right)$. Then,

$$
\begin{aligned}
\left|\mathrm{T}\left(\mathrm{x}_{i=1}^{n} \Sigma_{g_{i}},\left\{\bigoplus_{|\alpha|=p} \mathbf{h}_{\alpha_{1}, 1} \otimes \cdots \otimes \mathbf{h}_{\alpha_{n}, n}\right\}_{p=0}^{2 n}\right)\right| \\
=\prod_{i=1}^{n}\left|\mathrm{~T}\left(\Sigma_{g_{i}},\left\{\mathbf{h}_{p, i}\right\}_{p=0}^{2}\right)\right|^{\chi\left(\Sigma_{g_{1}}\right) \ldots \chi \widehat{\left(\Sigma_{g_{i}}\right) \ldots \chi\left(\Sigma_{g_{n}}\right)}},
\end{aligned}
$$

where $\times_{i=1}^{n} \Sigma_{g_{i}}$ is the cartesian product of $\Sigma_{g_{1}}, \ldots, \Sigma_{g_{n}}$, where $|\alpha|=\alpha_{1}+$ $\cdots+\alpha_{n}$ is the length of the multi-index $\alpha=\left(\alpha_{1}, \ldots, \alpha_{n}\right)$, and where in the product $\left.\chi\left(\Sigma_{g_{1}}\right) \ldots \chi \widehat{\left(\Sigma_{g_{i}}\right.}\right) \ldots \chi\left(\Sigma_{g_{n}}\right)$ is deletion of $\chi\left(\Sigma_{g_{i}}\right)$.

\subsection{Grassmannians and Schubert varieties}

We provide the basic definitions and necessary facts about the Grassmannians, Lagrangian Grassmannians, Orthogonal Grassmannians, and Isotropic Grass- 
mannians. For unexplained subject and further information, we refer the reader to [3], [4], [9], [10], [25]-[14], and the references therein.

Since the results corresponding to these manifolds are similar, we shall state for only one of them.

4.2.1. The Grassmannian $G(d, N)$. Let $E$ be $\mathrm{C}^{N}$ and let $G(d, E)=G(d, N)$ denote the Grassmannian of $d$-dimensional linear subspaces of $E$. This is a smooth algebraic variety of complex dimension $d n$, where $n=N-d$. It is well known that the Schubert cells stratify $G(d, N)$. The closures of these cells are called the Schubert varieties. More precisely, let $F_{\bullet}: 0=F_{0} \subset F_{1} \subset \cdots \subset$ $F_{N}=E$ be a complete flag of subspaces of $E$ with $\operatorname{dim} F_{i}=i, i=0, \ldots, N$. Let $\lambda=\left(\lambda_{1} \geq \lambda_{2} \geq \cdots \geq \lambda_{d} \geq 0\right)$ be a decreasing sequence of non-negative integers with $\lambda_{1} \leq n$. Then, the Young diagram of the partition $\lambda$ fits inside a $d \times n$ rectangle and this is denoted as $\lambda \subset\left(n^{d}\right)$.

The Schubert variety $X_{\lambda}\left(F_{\bullet}\right)$ associated to the complete flag $F_{\bullet}$ and the partition $\lambda$ is defined by

$$
X_{\lambda}\left(F_{\bullet}\right)=\left\{\Lambda \in G(d, N): \operatorname{dim}\left(\Lambda \cap F_{n+i-\lambda_{i}}\right) \geq i, i=1, \ldots, d\right\} .
$$

This is a codimension $|\lambda|$ closed subvariety of $G(d, N)$, where $|\lambda|=\sum \lambda_{i}$ is the weight of $\lambda$. By Poincare duality, $X_{\lambda}\left(F_{\bullet}\right)$ is associated to the Schubert class $\sigma_{\lambda}=\left[X_{\lambda}\left(F_{\bullet}\right)\right] \in H^{2|\lambda|}(G(d, N) ; \mathrm{Z})$. From the transitive action of $G L_{N}(\mathrm{C})$ on $G(d, N)$ and on the flags in $E$ it follows that $\sigma_{\lambda}$ is independent of the flag $F_{\bullet}$ used to define $X_{\lambda}$.

As an additive group $H^{*}(G(d, N) ; \mathrm{Z})=\bigoplus_{\lambda \subset\left(n^{d}\right)} \mathrm{Z} \cdot \sigma_{\lambda}$ is a free abelian group generated by the Schubert classes. Odd dimensional cohomologies are all zero and the Euler characteristic $\chi(G(d, N))=\left(\begin{array}{l}N \\ d\end{array}\right)$. Recall also that by the Schubert Duality theorem for any $\lambda$ and $\mu$ with $|\lambda|+|\mu|=d n$, we have $\int_{G(d, N)} \sigma_{\lambda} \sigma_{\mu}=\delta_{\hat{\lambda}, \mu}$, where $\hat{\lambda}=\left(\lambda_{N-d-\lambda_{d}}, \ldots, \lambda_{N-d-\lambda_{1}}\right)$ is the dual partition of $\lambda$.

From Theorem 0.1 it follows that

Theorem 4.6. Let $M=G(d, N)$ denote the Grassmannian of d-dimensional linear subspaces of $\mathrm{C}^{N}$. For $p=0, \ldots, 2 m$, let $\mathbf{h}_{p}$ be a basis of $H_{p}(M)$, where $m=d(N-d)$. Then, the following formulas hold:

(i) $\left|\mathrm{T}\left(M,\left\{\mathbf{h}_{p}\right\}_{0}^{2 m}\right)\right|=\prod_{p \in E_{m-1}}\left|\operatorname{det} H_{p, 2 m-p}(M)\right|$ for m odd,

(ii) $\left|\mathrm{T}\left(M,\left\{\mathbf{h}_{p}\right\}_{0}^{2 m}\right)\right|=\prod_{p \in E_{m-1}}\left|\operatorname{det} H_{p, 2 m-p}(M)\right| \sqrt{\left|\operatorname{det} H_{m, m}(M)\right|}$ for $m$ even,

where $E_{m-1}$ is the set of even numbers in $\{0, \ldots, m-1\}$.

In particular, let us consider the complex projective space $\mathrm{CP}^{m}$. For $p$ even $H^{p}\left(\mathrm{CP}^{m}\right)$ is generated by $\omega_{\mathrm{FS}}^{p}$, where $\omega_{\mathrm{FS}}$ is the Fubini-Study metric of $\mathrm{CP}^{m}$ 
and $\omega_{\mathrm{FS}}^{p}$ denotes the $p$ times wedge product of $\omega_{\mathrm{FS}}$. Using also the Poincaré Duality, we have

CoROllary 4.7. If for $p=0, \ldots, 2 m, \mathbf{h}^{p}$ is a basis of $H^{p}\left(\mathrm{CP}^{m}\right)$, then

(i) $\left|\mathrm{T}\left(\mathrm{CP}^{m},\left\{\mathbf{h}_{p}\right\}_{0}^{2 m}\right)\right|=V_{m} \prod_{p \in E_{m-1}}\left|\lambda_{p}\right|\left|\lambda_{2 m-p}\right|$ for $m$ odd,

(ii) $\left|\mathrm{T}\left(\mathrm{CP}^{m},\left\{\mathbf{h}_{p}\right\}_{0}^{2 m}\right)\right|=V_{m} \prod_{p \in E_{m-1}}\left|\lambda_{p}\right|\left|\lambda_{2 m-p}\right|\left|\lambda_{m}\right|$ for $m$ even,

where $\mathbf{h}_{p} \in H_{p}\left(\mathrm{CP}^{m}\right)$ is the Poincaré dual of $\mathbf{h}^{p} \in H^{p}\left(\mathrm{CP}^{m}\right)$ and $\mathbf{h}^{p}=\lambda_{p} \omega_{\mathrm{FS}}^{p}$ for some $\lambda_{p} \in \mathrm{R}$, where $\omega_{\mathrm{FS}}$ is the Fubuni-Study form of $\mathrm{CP}^{m}$, where $E_{m-1}$ is the set of even numbers in $\{0, \ldots, m-1\}$, and where $V_{m}=\left(\frac{1}{m !} \operatorname{Vol}\left(\mathrm{CP}^{m}\right)\right)^{\chi\left(\mathrm{CP}^{m}\right) / 2}$.

We would like to conclude this section with the following example.

ExAmple 4.8. Let $M=\mathrm{CP}^{3}$ and $N=\mathrm{CP}^{6}$. Using the Künneth formula, we get $H_{0}(M \times N)=H_{0}(M) \otimes H_{0}(N), H_{18}(M \times N)=H_{6}(M) \otimes H_{12}(N)$, $H_{2}(M \times N)=H_{0}(M) \otimes H_{2}(N) \oplus H_{2}(M) \otimes H_{0}(N), H_{16}(M \times N)=H_{6}(M) \otimes$ $H_{10}(N) \oplus H_{4}(M) \otimes H_{12}(N), H_{4}(M \times N)=H_{0}(M) \otimes H_{4}(N) \oplus H_{2}(M) \otimes$ $H_{2}(N) \oplus H_{4}(M) \otimes H_{0}(N), H_{14}(M \times N)=H_{6}(M) \otimes H_{8}(N) \oplus H_{4}(M) \otimes$ $H_{10}(N) \oplus H_{2}(M) \otimes H_{12}(N), H_{6}(M \times N)=H_{0}(M) \otimes H_{6}(N) \oplus H_{2}(M) \otimes$ $H_{4}(N) \oplus H_{4}(M) \otimes H_{2}(N) \oplus H_{6}(M) \otimes H_{0}(N), H_{12}(M \times N)=H_{6}(M) \otimes$ $H_{6}(N) \oplus H_{4}(M) \otimes H_{8}(N) \oplus H_{2}(M) \otimes H_{10}(N) \oplus H_{0}(M) \otimes H_{12}(N), H_{8}(M \times$ $N)=H_{0}(M) \otimes H_{8}(N) \oplus H_{2}(M) \otimes H_{6}(N) \oplus H_{4}(M) \otimes H_{4}(N) \oplus H_{6}(M) \otimes$ $H_{2}(N)$, and $H_{10}(M \times N)=H_{6}(M) \otimes H_{4}(N) \oplus H_{4}(M) \otimes H_{6}(N) \oplus H_{2}(M) \otimes$ $H_{8}(N) \oplus H_{0}(M) \otimes H_{10}(N)$.

From these it follows that

$$
\begin{aligned}
& \left|H_{4,14}(M \times N)\right| \\
& \quad=\left|H_{0,6}(M)\right|\left|H_{2,4}(M)\right|^{2}\left|H_{0,12}(N)\right|\left|H_{2,10}(N)\right|\left|H_{4,8}(N)\right|,
\end{aligned}
$$

$$
\begin{aligned}
& \left|H_{6,12}(M \times N)\right| \\
& \quad=\left|H_{0,6}(M)\right|^{2}\left|H_{2,4}(M)\right|^{2}\left|H_{0,12}(N)\right|\left|H_{2,10}(N)\right|\left|H_{4,8}(N)\right|\left|H_{6,6}(N)\right|,
\end{aligned}
$$

(4.20) $\left|H_{8,10}(M \times N)\right|$

$$
=\left|H_{0,6}(M)\right|^{2}\left|H_{2,4}(M)\right|^{2}\left|H_{2,10}(N)\right|\left|H_{4,8}(N)\right|^{2}\left|H_{6,6}(N)\right| .
$$

Combining (4.16)-(4.20), we obtain that $|\mathrm{T}(M \times N)|=$ $|\mathrm{T}(M)|^{\chi(N)}|\mathrm{T}(N)|^{\chi(M)}$. 
4.2.2. The Lagrangian Grassmannian $L G(n, 2 n)$. Let $E$ be $\mathrm{C}^{2 n}$ equipped with a symplectic form $\langle\cdot, \cdot\rangle$. A subspace $V$ of $E$ is isotropic if the restriction of the symplectic form $\langle\cdot, \cdot\rangle$ to $V$ vanishes. Note that the maximal possible dimension of an isotropic subspace is $n$, and in this case $V$ is called a Lagrangian subspace of $E$. The Lagrangian Grassmannian $L G(n, 2 n)$ is a complex manifold of complex dimension $n(n+1) / 2$ parametrizing the Lagrangian subspaces in $E$.

A complete isotropic flag $F_{\bullet}: 0=F_{0} \subset F_{1} \subset \cdots \subset F_{n} \subset E$ of subspaces of $E$ is a flag of isotropic subspaces of $E$ such that $\operatorname{dim} F_{i}=i$ for each $i$. Thus, a complete isotropic flag is a Lagrangian subspace $F_{n}$ of $E$ together with a complete flag of subspaces of $F_{n}$. In fact, any isotropic flag $F_{\bullet}: 0=F_{0} \subset F_{1} \subset \cdots \subset F_{n} \subset E$ can be completed to a complete flag by setting $F_{n+i}=F_{n-i}^{\perp}, i=1, \ldots, n$.

Let $F_{\bullet}$ be a complete isotropic flag of $E$ and $\lambda=\left(\lambda_{1}>\lambda_{2}>\cdots>\lambda_{\ell}>0\right)$ with $\lambda_{1} \leq n$ be a strictly decreasing partition. The codimension $|\lambda|=\sum \lambda_{i}$ Schubert variety $X_{\lambda}\left(F_{\bullet}\right) \subset L G(n, 2 n)$ is defined by

$$
X_{\lambda}\left(F_{\bullet}\right)=\left\{\Lambda \in L G(n, 2 n): \operatorname{dim}\left(\Lambda \cap F_{n+1-\lambda_{i}}\right) \geq i, i=1, \ldots, \ell(\lambda)\right\},
$$

where $\ell(\lambda)$ is the length of $\lambda$, i.e., the number of non-zero terms in $\lambda$.

Let $\sigma_{\lambda}=\left[X_{\lambda}\left(F_{\bullet}\right)\right] \in H^{2|\lambda|}(L G(n, 2 n) ; \mathrm{Z})$ be the cohomology class of $X_{\lambda}\left(F_{\bullet}\right) . H^{*}(L G(n, 2 n) ; \mathrm{Z})$ is a free abelian group generated by the Schubert classes $\sigma_{\lambda}$ with strictly decreasing partition $\lambda$. Recall the Poincaré duality $\int_{L G(n, 2 n)} \sigma_{\lambda} \sigma_{\mu}=\delta_{\check{\lambda}, \mu}$, where $\check{\lambda}=\rho_{n}-\lambda$ is the dual partition of $\lambda$, and where $\rho_{n}=(n, n-1, \ldots, 1)$. Recall also that the Euler characteristic of $L G(n, 2 n)$ is $2^{n}$.

Moreover, for $L G(n, 2 n)$, we have a result similar to Theorem 4.6, where $m=n(n+1) / 2$.

4.2.3. The Orthogonal Grassmannian $O G(n+1,2 n+2)$. Let $E$ be $C^{2 n+2}$ equipped with a non-degenerate symmetric form. The even orthogonal Grassmannian $O G(n+1,2 n+2)$ parametrizes one component of the locus of maximal isotropic subspaces of $E$. This is a complex manifold of complex dimension $n(n+1) / 2$. There are two families of such subspaces. As convention, given a fixed isotropic flag $F_{\bullet}$ in $E$, only those isotropic $\Lambda$ in $E$ with $\Lambda \cap F_{n+1}$ even codimension in $F_{n+1}$ are considered. Recall that $O G(n+1,2 n+2)$ is isomorphic to the odd Orthogonal Grassmannian $O G(n, 2 n+1)$.

As in $L G(n, 2 n)$, the Schubert varieties $X_{\lambda}\left(F_{\bullet}\right)$ in $O G(n+1,2 n+2)$ are also parametrized by strictly decreasing partitions $\lambda=\left(\lambda_{1}>\lambda_{2}>\cdots>\lambda_{\ell}>0\right)$ with $\lambda_{1} \leq n$ and defined by

$$
X_{\lambda}\left(F_{\bullet}\right)=\left\{\Lambda \in O G(n+1,2 n+2): \operatorname{dim}\left(\Lambda \cap F_{n+1-\lambda_{i}}\right) \geq i, i=1, \ldots, \ell(\lambda)\right\}
$$


with respect to a complete isotropic flag $F_{0}$ in $E$. Let $\sigma_{\lambda}$ be the cohomology class of $X_{\lambda}\left(F_{\bullet}\right)$. The abelian group $H^{*}(O G(n+1,2 n+2)$; Z) is generated by the Schubert classes $\sigma_{\lambda}$ with strictly decreasing partition $\lambda$. Moreover, $\chi(O G(n+1,2 n+2))=2^{n}$.

Similar result of Theorem 4.6 also holds for $O G(n+1,2 n+2)$, where $m=n(n+1) / 2$.

4.2.4. The Grassmannian $I G(n-k, 2 n)$. Let us fix a vector space $E \cong \mathrm{C}^{2 n}$ with a non-degenerate skew-symmetric bilinear form $\langle\cdot, \cdot\rangle$, and let $d \leq n$ be a fixed non-negative integer. The Isotropic Grassmannian $I G(d, 2 n)$ parametrizes $d$ dimensional isotropic subspaces of $E$. This is an algebraic variety of complex dimension $2 d(n-d)+d(d+1) / 2$.

Let $k$ be a non-negative integer. The partition $\lambda$ is said to be $k$-strict, if no part of $\lambda$ greater than $k$ is repeated, namely $\lambda_{i}>k \Rightarrow \lambda_{i+1}<\lambda_{i}$.

Now, let $k=n-d$. The Schubert varieties in $I G(d, 2 n)$ are parametrized by the set $\mathscr{P}(k, n)$ of all $k$-strict partitions contained in a $d \times(n+k)$ rectangle.

Recall that an isotropic flag in $E$ is a complete flag $F_{\bullet}: 0=F_{0} \subset F_{1} \subset$ $\cdots \subset F_{2 n}=E$ of subspaces such that $F_{n+i}=F_{n-i}^{\perp}, i=0, \ldots, n$. For each $\lambda \in \mathscr{P}(k, n)$, the Schubert variety relative to the isotropic flag $F_{\bullet}$ is

$$
X_{\lambda}\left(F_{\bullet}\right)=\left\{\Lambda \in I G(d, 2 n): \operatorname{dim}\left(\Lambda \cap F_{p_{j}(\lambda)}\right) \geq j, j=1, \ldots, \ell(\lambda)\right\},
$$

where $p_{j}(\lambda)=n+k+1-\lambda_{j}+\sharp\left\{i<j: \lambda_{i}+\lambda_{j} \leq 2 k+j-i\right\}$, and where $\ell(\lambda)$ is the length of $\lambda$.

This is a codimension $|\lambda|$ variety. Let $\sigma_{\lambda}$ denote $\left[X_{\lambda}\right] \in H^{2|\lambda|}(I G(d, 2 n) ; Z)$. The cohomology ring $H^{*}(I G(d, 2 n) ; Z)$ is a free abelian group generated by these Schubert classes. Moreover, the $k$-strict partition $\lambda$ has a unique dual partition $\check{\lambda} \in \mathscr{P}(k, n)$, for which $p_{j}(\check{\lambda})=2 n+1-p_{d+1-j}(\lambda), j=1, \ldots, d$. We also have $\int_{I G(d, 2 n)} \sigma_{\lambda} \sigma_{\mu}=\delta_{\mu, \check{\lambda}}$. Finally, the Euler characteristic of $\operatorname{IG}(d, 2 n)$ is the rank of $H^{*}(I G(d, 2 n) ; Z)=\sharp \mathscr{P}(k, n)=2^{d}\left(\begin{array}{l}n \\ k\end{array}\right)$.

For $I G(d, 2 n)$, we also obtain a result similar to Theorem 4.6 where $m=$ $2 d(n-d)+d(d+1) / 2$.

4.2.5. The Grassmannian $O G(n-k, 2 n+1)$. Let $E \cong \mathrm{C}^{2 n+1}$ be a vector space with a non-degenerate symmetric bilinear form on $E$. For $d=n-k<n$, let $O G(d, 2 n+1)$ denote the Odd Orthogonal Grassmannian parametrizing the $d$-dimensional isotropic subspaces of $E$. Like $I G(d, 2 n)$, the algebraic variety $O G(n-k, 2 n+1)$ has also complex dimension $2 d(n-d)+d(d+1) / 2$. Furthermore, as in $I G(d, 2 n)$, the Schubert varieties are parametrized by the set of $k$-strict partitions $\mathscr{P}(k, n)$.

Recall that an isotropic flag $F_{\bullet}$ is a complete flag $0=F_{0} \subset F_{1} \subset \cdots \subset$ $F_{2 n+1}=E$ such that $F_{n+i}=F_{n+1-i}^{\perp}, i=1, \ldots, n+1$. Let $F_{\bullet}$ be an isotropic 
flag and let $\lambda \in \mathscr{P}(k, n)$. The Schubert variety associated to $F_{\bullet}$ and $\lambda$ is

$$
X_{\lambda}\left(F_{\bullet}\right)=\left\{\Lambda \in O G(d, 2 n+1): \operatorname{dim}\left(\Lambda \cap F_{\bar{p}_{j}(\lambda)}\right) \geq j, j=1, \ldots, \ell(\lambda)\right\},
$$

where $\bar{p}_{j}(\lambda)=p_{j}(\lambda)+\mathbf{1}_{\{0, \ldots, k\}}\left(\lambda_{j}\right)$, where $p_{j}(\lambda)=n+k+1-\lambda_{j}+\sharp\{i<$ $\left.j: \lambda_{i}+\lambda_{j} \leq 2 k+j-i\right\}$, and where

$$
\mathbf{1}_{\{0, \ldots, k\}}\left(\lambda_{j}\right)= \begin{cases}1, & \lambda_{j} \leq k \\ 0, & \lambda_{j}>k\end{cases}
$$

This variety has codimension $|\lambda|$. Let $\sigma_{\lambda}$ denote the cohomology class of Poincaré dual to the cycle given by $X_{\lambda}\left(F_{\bullet}\right)$. The abelian group $H^{*}(O G(d, 2 n+$ $1)$; Z) is generated by these Schubert classes. We also have $\int_{O G(d, 2 n+1)} \sigma_{\lambda} \sigma_{\mu}=$ $\delta_{\mu, \check{\lambda}}$, where $\bar{p}_{j}(\check{\lambda})=2 n+2-p_{d+1-j}(\lambda)$. The rank of $H^{*}(O G(d, 2 n+1) ; Z)$ is equal to the rank of $H^{*}\left(I G(n-k, 2 n)\right.$; Z), i.e., $\sharp \mathscr{P}(k, n)=2^{d}\left(\begin{array}{l}n \\ k\end{array}\right)$.

Furthermore, for $O G(d, 2 n+1)$, there is a result similar to Theorem 4.6, where $m=2 d(n-d)+d(d+1) / 2$.

4.2.6. The Grassmannian $O G(n+1-k, 2 n+2)$. Let $E \cong \mathrm{C}^{2 n+2}$ be a vector space with a non-degenerate symmetric bilinear form on $E$. For $d=$ $n+1-k<n$, let $O G(d, 2 n+2)$ be the even Orthogonal Grassmannian parametrizing the $d$-dimensional isotropic subspaces of $E$. This is a variety of complex dimension $2 d(n+1-d)+d(d-1) / 2$.

The subspaces $U, V$ of $E$ are in the same family if $\operatorname{dim}(U \cap V) \equiv(n+$ 1) $(\bmod 2)$. Fix an isotropic subspace $W$ of $E$ with $\operatorname{dim} W=n+1$. An isotropic flag is a complete flag $F_{\text {. }}$ of subspaces of $E$ such that $F_{n+1+i}=F_{n+1-i}^{\perp}$, $i=0, \ldots, n$, and $F_{n+1}$ and $W$ are in the same family. Since the orthogonal space $F_{n}^{\perp} / F_{n}$ contains only two isotropic lines, to each such flag $F_{0}$, there is an alternate isotropic flag $\tilde{F}_{\text {}}$ such that for $i \leq n \tilde{F}_{i}=F_{i}$ but with $\tilde{F}_{n+1}$ in the opposite family from $F_{n+1}$.

Let $k=n+1-d>0$. The $k$-strict partition $\lambda$ is of type 0 if it has no part equal to $k$. Otherwise, $\lambda$ is of type 1 or 2 . Type is a multi-valued function. Let $\tilde{\mathscr{P}}(k, n)$ be the set of all $k$-strict partitions contained in a $d \times(n+k)$ rectangle of all three possible types. For $\lambda \in \tilde{\mathscr{P}}(k, n)$, let us define an index set $P^{\prime}=\left\{p_{1}^{\prime}<\cdots<p_{d}^{\prime}\right\} \subset\{1, \ldots, 2 n+2\}$ with

$$
\begin{aligned}
p_{j}^{\prime}(\lambda)=n+ & k-\lambda_{j}+\sharp\left\{i<j: \lambda_{i}+\lambda_{j} \leq 2 k-1+j-i\right\} \\
& + \begin{cases}1, & \lambda_{j}>k \text { or } \lambda_{j}=k<\lambda_{j-1} \text { and } n+j+\operatorname{type}(\lambda) \text { is even } \\
2, & \text { otherwise. }\end{cases}
\end{aligned}
$$

Let $F$. be an isotropic flag. For each $\lambda \in \tilde{\mathscr{P}}(k, n)$, the codimension $|\lambda|$ Schubert variety is $X_{\lambda}\left(F_{\bullet}\right)=\left\{\Lambda \in O G(d, 2 n+2): \operatorname{dim}\left(\Lambda \cap F_{p_{j}^{\prime}(\lambda)}\right) \geq\right.$ 
$j$, if $p_{j}^{\prime} \neq n+2, \operatorname{dim}\left(\Lambda \cap \tilde{F}_{n+1}\right) \geq j$, if $p_{j}^{\prime}=n+2$, for all $\left.j=1, \ldots, \ell(\lambda)\right\}$. Let $\sigma_{\lambda}$ be the cohomology class in $H^{2|\lambda|}(O G(d, 2 n+2)$; Z) Poincaré dual to the cycle determined by the Schubert variety associated to $\lambda$. The free abelian group $H^{*}(O G(d, 2 n+2) ; \mathrm{Z})$ is generated by the Schubert classes and the rank of $H^{*}(O G(d, 2 n+2) ; \mathrm{Z})$ is $2^{n+1-k}\left(\begin{array}{c}n+1 \\ k\end{array}\right)$. For each $\lambda \in \tilde{\mathscr{P}}(k, n)$, define a dual partition $\check{\lambda} \in \tilde{\mathscr{P}}(k, n)$ by

$$
p_{j}^{\prime}(\check{\lambda})= \begin{cases}2 n+3-p_{d+1-j}^{\prime}(\lambda), & \text { if } n \text { is odd or } p_{j}^{\prime}(\lambda) \neq n+1, n+2 \\ p_{j}^{\prime}(\lambda), & \text { if } n \text { is even and } p_{j}^{\prime}(\lambda) \in\{n+1, n+2\} .\end{cases}
$$

Moreover, for $\lambda, \mu \in \tilde{\mathscr{P}}(k, n)$, we have $\int_{O G(d, 2 n+2)} \sigma_{\lambda} \sigma_{\mu}=\delta_{\mu, \check{\lambda}}$.

For $O G(d, 2 n+2)$, similar result as Theorem 4 .6 holds, where $m=2 d(n+$ $1-d)+d(d-1) / 2$.

AcKnowledgement. We are indepted to the reviewer for his/her careful reading and constructive criticism which improved the content of this paper.

\section{REFERENCES}

1. Bismut, J. M., Gillet, H., and Soulé, C., Analytic torsion and holomorphic determinant bundles I. Bott-Chern forms and analytic torsion, Comm. Math. Phys. 115 (1988), 49-78.

2. Bismut, J. M., Labourie, F., Symplectic geometry and the Verlinde formulas, pp. 97-311 in: S. T. Yau (ed.), Surveys in Differential Geometry: Differential Geometry Inspired by String Theory, Surv. Differ. Geom. 5, Int. Press, Boston 1999.

3. Buch, A. S., Kresch, A., and Tamvakis, H., Gromov-Witten invariants on Grassmannians, J. Amer. Math. Soc. 16 (2003), 901-915.

4. Buch, A. S., Kresch, A., and Tamvakis, H., Quantum Pieri rules for isotropic Grassmannians, Invent. Math. 178 (2009), 345-405.

5. Chapman, T. A., Compact Hilbert cube manifolds and the invariance of Whitehead torsion, Bull. Amer. Math. Soc. 79 (1973), 52-56.

6. Chapman, T. A., Topological invariance of Whitehead torsion, Amer. J. Math. 96 (1974), 488-497.

7. de Rham, G., Reidemeister's torsion invariant and rotation of $S^{n}$, pp. 27-36 in: Differential Analysis, Tata Institute and Oxford Univ. Press, Oxford 1964.

8. Franz, W., Über die Torsion einer Überdeckung, J. Reine Angew. Math. 173 (1935), 245-254.

9. Fulton, W., and Pragacz, P., Schubert Varieties and Degeneracy Loci, Lecture Notes in Math. 1689, Springer, Berlin 1998.

10. Griffiths, P., and Harris, J., Principles of Algebraic Geometry, Wiley, New York 1994.

11. Hatcher, A., Algebraic Topology, Cambridge Univ. Press, Cambridge 2002.

12. Kirby, R. C., Siebenmann, L. C., On triangulation of manifolds and Haupvermutung, Bull. Amer. Math. Soc. 75 (1969), 742-749.

13. Kresch, A., and Tamvakis, H., Quantum cohomology of the Lagrangian Grassmannian, J. Algebraic Geom. 12 (2003), 777-810.

14. Kresch, A., and Tamvakis, H., Quantum cohomology of orthogonal Grassmannians, Compos. Math. 140 (2004), 482-500.

15. May, J. P., A Concise Course in Algebraic Topology, Univ. of Chicago Press, Chicago 1999. 
16. Milnor, J., A duality theorem for Reidemeister torsion, Ann. of Math. (2) 76 (1962), 137-147.

17. Milnor, J., Whitehead torsion, Bull. Amer. Math. Soc. 72 (1966), 358-426.

18. Milnor, J., Infinite cyclic coverings, pp. 115-133 in: Conference on the Topology of Manifolds, Proc. East Lansing 1967, Prindle, Weber \& Schmidt, Boston 1968.

19. Porti, J., Torsion de Reidemeister pour les variétés hyperboliques, Mem. Amer. Math. Soc. 128 (1997), no. 612.

20. Reidemeister, K., Homotopieringe und Linsenräume, Abh. Math. Sem. Univ. Hamburg 11 (1935), 102-109.

21. Shilov, G. E., Linear Algebra, Dover, New York 1977.

22. Sözen, Y., Reidemeister torsion of a symplectic complex, Osaka J. Math. 45 (2008), 1-39.

23. Sözen, Y., On Fubini-Study form and Reidemeister torsion, Topology Appl. 156 (2009), 951955.

24. Sözen, Y., A note on Reidemeister torsion and period matrix of Riemann surfaces, Math. Slovaca 61 (2011), 29-38.

25. Tamvakis, H., Quantum cohomology of isotropic Grassmannians, pp. 311-338 in: Geometric Methods in Algebra and Number Theory, Progr. Math. 235, Birkhäuser, Boston 2005.

26. Tamvakis, H., Gromov-Witten invariants and quantum cohomology of Grassmannians, pp. 271-297 in: Topics in Cohomological Studies of Algebraic Varieties, Trends in Math., Birkhäuser, Basel 2005.

27. Witten, E., On quantum gauge theories in two dimensions, Comm. Math. Phys. 141 (1991), 153-209.

FATIH UNIVERSITY

BÜYÜKÇEKMECE

ISTANBUL

TURKEY

E-mail: ysozen@fatih.edu.tr 